\title{
The effective $\sigma$-model of multidimensional gravity
}

\author{
Martin Rainer* \\ Center for Gravitational Physics and Geometry, \\ 104 Davey Laboratory, The Pennsylvania State University, \\ University Park, PA 16802-6300, USA \\ and \\ Gravitationsprojekt, Mathematische Physik I, \\ Institut für Mathematik,Universität Potsdam, \\ PF 601553, D-14415 Potsdam, Germany
}

October 1998

\begin{abstract}
The properties of the effective $\sigma$-model for $D$-dimensional Einstein gravity based on multidimensional geometries is analyzed. Besides pure geometry additional minimally coupled scalars and $(p+2)$-forms are considered which yield an extended target space after reduction to the effective $D_{0}$-dimensional geometry.

The target space is always a homogeneous space. Exact solutions exist provided an orthobrane condition is satisfied which geometrically makes the target space a locally symmetric one.

New solutions with scalar fields are found which may inflate not only in time-like but in also in additional spatial directions of the effective geometry.

Static spherically symmetric solutions with a particular configuration of intersecting electric and magnetic branes are investigated both, for the orthobrane case and for degenerated charges. In both cases $T_{H}$ depends critically on the intersection dimension of the branes.

Finally, the role of the Einstein frame for 4-geometries is addressed, and the physical frame transformation for cosmological geometries is given.
\end{abstract}

PACS: 04.50.+h, 04.70.-s, 02.40.Hw

*E-mail: rainer@phys.psu.edu 


\section{Introduction}

Historically $\sigma$-models have turned out to be a very powerful tool in many areas of physics. In gravity the importance was soon realized [1] in the context of solution generating techniques 2]. More recently, $\sigma$-models have been also discussed in the context of string theory [3, 4, 5].

The purpose of this paper is to clarify the geometric structure of the effective $\sigma$-model for multidimensional Einstein geometry and to demonstrate its applicability in such different directions as cosmology, (extended) string theory, and quantization of certain higher-dimensional geometric actions.

In fact it turns out to be a very powerful tool which, on one side, allows to test the geometric content of string and M-theory down to their concrete physical imprints in the physical space-time and, on the other side, prepares a well defined class of classical higher-dimensional geometries for the canonical quantization program in dimension $D_{0} \leq 4$ whenever this is applicable to pure Einstein gravity itself. In principle all cases with infinite number of degrees of freedom in dimension $D_{0}=4$ which can be canonically quantized have some analogous cases where additional extra dimensions add only a finite number of degrees of freedom without disturbing the integrability of the problem. These cases include of course also recently investigated midisuperspace 4-geometries. In the case of spherical symmetries, and more particular in the static case, one can find particular solutions to a classical system of the multidimensional Einstein action with scalar and antisymmetric $p+2$-form fields which are multidimensional extensions of black hole solutions. It turns out that the standard surface gravity and the Hawking temperature $T_{H}$ as calculated from a Komar-like integral depend sensitively on the intersection dimension of the $p$-branes involved in the solution. This provides, at least in principle, an observational window to very direct geometrical properties of possible extra dimensions. Apart from that, the multidimensional $\sigma$-model contains all kinds of multidimensional spatially homogeneous cosmological models as degenerate minisuperspace cases with a finite number of degrees of freedom only.

Below, the effective $D_{0}$-dimensional $\sigma$-model is derived from a multidimensional action of Einstein type in a higher dimension $D$, first for pure geometry, then with additional scalar and antisymmetric $p+2$-form matter fields. The domains of the $p+1$-form potentials of the antisymmetric $p+2$-forms are the world-sheets of $p$-branes. In extended string and Mtheory [6, 7, 8] strings are generalized to membranes as higher-dimensional objects. Most of these unified models are modeled initially on a higher-dimensional space-time manifold, say of dimension $D>4$, which then undergoes some scheme of spontaneous compactification.

The geometric structure of the target-space is clarified. In particular it is shown that it is a always a homogeneous space. It is furthermore locally symmetric if and only if the characteristic target-space vectors satisfy a particular orthogonality condition, called the orthobrane relation 
whenever they are not identical. In any case, it turns out possible to express the general exact solutions in terms of elementary functions, provided the input parameters of the model satisfy the, whence the target space is locally symmetric.

Solutions of the corresponding field equations are discussed generally and with concrete examples. Particular solutions for the subcases with Ricci flat internal spaces with scalar fields only, and with intersecting p-branes are presented. In the subcase of spherically symmetric solutions the relation to particles and black $p$-branes is given. Although a priori one might admit all possible types of components of $F$-fields compatible with spherical symmetry, namely, electric, magnetic and quasiscalar ones, we concentrate on true electric and magnetic type fields, since these are the ones which admit black hole solutions.

Besides the orthobrane solutions which by now became popular in string theory, there are further families of solutions, which have another additional symmetry, e.g. coinciding $F$-field charges for the electro-magnetic solutions. In target space this additional symmetry is expressed by a linear relation between certain column vectors of the coupling matrix. In this case the original orthobrane conditions reduce to some weaker set of orthogonality conditions.

In the case of static, spherical symmetric solutions it is demonstrated that the formal Hawking temperature $T_{H}$ (as it might appear to an observer at infinity) depends sensitively on the intersection dimension of the $p$-branes. Hence solutions to the multidimensional $\sigma$-model allow to detect possible imprints from extra-dimensional internal factor spaces within the physical dimension $D_{0}=4$. The black hole solutions depend on 3 integration constants, related to the electric, the magnetic, and the mass charge. It is also shown that the Hawking temperature of such black holes depends on the intersection dimension $d_{\text {int }}$ of the corresponding $p$-branes. In an extremal limit of the charges, the black hole temperature turns out to converge to zero for $d_{\text {int }}=0$, to a finite limit for $d_{\text {int }}=1$, and to infinity for $d_{\text {int }}>1$.

Finally it is shown how the geometries of well known solutions in a Brans-Dicke frame can be transformed to the physically relevant Einstein frame.

\section{Pure multidimensional gravity}

For the purpose of this paper let a $\left(C^{\infty}\right)$ multidimensional (MD) manifold $N$ be topologically just defined by a $C^{\infty}$-fiber bundle

$$
M \hookrightarrow N \rightarrow \bar{M}_{0}
$$

with a direct product

$$
M:=\times_{i=1}^{n} M_{i}
$$

of internal $C^{\infty}$ factor spaces $M_{i}, i=1, \ldots, n$, as a standard fiber, and a distinguished $C^{\infty}$ base

manifold $\bar{M}_{0}$. (Later, for considerations of dynamics and cosmology we will set in particular 
$\bar{M}_{0}:=\mathbb{R} \times M_{0}$, and for the connection representation of Einstein gravity $D_{0}:=4$ will be required.)

The MD manifold $N$ is called internally homogeneous if there exists a direct product group $G:=\bigotimes_{i=0}^{n} G_{i}$ with a direct product realization $\tau:=\bigotimes_{i=0}^{n} \tau_{i}$ on $\operatorname{Diff}(M):=\bigotimes_{i=0}^{n} \operatorname{Diff}\left(M_{i}\right)$ such that for $i=0, \ldots, n$ the realization

$$
\tau_{i}: G_{i} \rightarrow \operatorname{Diff}\left(M_{i}\right)
$$

yields a transitive action of $\tau_{i}\left(G_{i}\right)$ on $M_{i}$.

Definition: A $\left(C^{\infty}\right)$ Riemannian manifold $(M, g)$ (of arbitrary signature) is a $C^{\infty}$ manifold $M$ equipped with a symmetric bilinear $C^{\infty}$ section $g: M \rightarrow \mathfrak{T}_{2}^{0} M$ called metric. Unless specified otherwise the metric $g$ will always be assumed to be non-degenerate.

Definition: Given a Riemannian manifold $(M, g)$, a diffeomorphism $\chi \in \operatorname{Diff}(M)$ is called an isometry of $(M, g)$ whenever it leaves $g$ invariant, i.e. whenever

$$
g_{\chi(p)}=g_{p} \quad \forall p \in M .
$$

The very fact that a given diffeomorphism $\chi \in \operatorname{Diff}(M)$ may be an isometry on some metric but not on another one is the reason why the action of $\operatorname{Diff}(M)$ is not free on the space $\operatorname{Met}(M)$ of $C^{\infty}$-metrics on $M$, whence $\operatorname{Geom}(M):=\operatorname{Met}(M) / \operatorname{Diff}(M)$ is in general not a manifold.

Definition: A Riemannian manifold $(M, g)$ is called homogeneous, whenever $M$ is homogeneous with a corresponding group $G$ having a transitive realization $\tau(G) \subset \operatorname{Diff}(M)$ which leaves $g$ invariant, i.e.

$$
g_{\chi(p)}=g_{p} \quad \forall p \in M \quad \forall \chi \in \tau(G) .
$$

Now for $i=0, \ldots, n$, let each factor space $M_{i}$ be equipped with a smooth homogeneous metric $g^{(i)}$. rendering it into a homogeneous Riemannian manifold. Furthermore, let $\bar{M}_{0}$ be equipped with an arbitrary $C^{\infty}$-metric $\bar{g}^{(0)}$, and let $\bar{\gamma}$ and $\beta^{i}, i=1, \ldots, n$ be smooth scalar fields on $\bar{M}_{0}$.

Then, under any projection pr $: N \rightarrow \bar{M}_{0}$ a pullback of $e^{2 \bar{\gamma}} \bar{g}^{(0)}$ from $x \in \overline{M_{0}}$ to $z \in$ $\operatorname{pr}^{-1}\{x\} \subset M$, consistent with the fiber bundle (2.1) and the homogeneity of internal spaces, is given by

$$
g_{(z)}:=e^{2 \bar{\gamma}(x)} \bar{g}_{(x)}^{(0)} \oplus_{i=1}^{n} e^{2 \beta^{i}(x)} g^{(i)} .
$$


The function $\bar{\gamma}$ fixes a gauge for the (Weyl) conformal frame on $\overline{M_{0}}$, corresponding just to a particular choice of geometrical variables.

$\bar{\gamma}$ uniquely defines the form of the effective $D_{0}$-dimensional theory. For example $\bar{\gamma}:=0$ defines the Brans-Dicke frame,

Let us now consider a multidimensional manifold $N$ (2.1) of dimension $D=D_{0}+\sum_{i=0}^{n} d_{i}$, equipped with a (pseudo) Riemannian metric (2.6) where

$$
g^{(i)} \equiv g_{m_{i} n_{i}}\left(y_{i}\right) d y_{i}^{m_{i}} \otimes d y_{i}^{n_{i}}
$$

are $R$-homogeneous Riemannian metrics on $M_{i}$ (i.e. the Ricci scalar $R\left[g^{(i)}\right] \equiv R_{i}$ is a constant on $M_{i}$ ), in coordinates $y_{i}^{n_{i}}, n_{i}=0, \ldots, d_{i}$, and

$$
x \mapsto \bar{g}^{(0)}(x)=\bar{g}_{\mu \nu}^{(0)}(x) d x^{\mu} \otimes d x^{\nu}
$$

yielding a general, not necessarily $R$-homogeneous, (pseudo) Riemannian metric on $\bar{M}_{0}$.

With (2.6) is a multidimensional generalization of the warped product of [9], namely $N=$ $\bar{M}_{0} \times{ }_{a} M$, where $a:=e^{\beta}$ is now a vector-valued root warping function, given by

$$
\beta:=\left(\begin{array}{l}
\beta_{0} \\
\vdots \\
\beta_{n}
\end{array}\right) .
$$

Below sometimes, in particular for physical application to the $\operatorname{Diff}\left(\bar{M}_{0}\right)$-invariant case with $D_{0}=4$, we will assume the $i=0$ geometry to be empty and omit corresponding empty contributions to tensors, summations etc. For later convenience we also define

$$
\varepsilon(I):=\prod_{i \in I} \varepsilon_{i} ; \quad \sigma_{0}:=\sum_{i=0}^{n} d_{i} \beta_{i}, \quad \sigma_{1}:=\sum_{i=1}^{n} d_{i} \beta_{i}, \quad \sigma(I):=\sum_{i \in I} d_{i} \beta_{i},
$$

where $\varepsilon_{i}:=\operatorname{sign}\left(\left|g^{(i)}\right|\right)$ and $M_{i} \subset M$ for $i=0, \ldots, n$ are all homogeneous factor spaces. Here and below, we use the shorthand $|g|:=\left|\operatorname{det}\left(g_{M N}\right)\right|,\left|\bar{g}^{(0)}\right|:=\left|\operatorname{det}\left(\bar{g}_{\mu \nu}^{(0)}\right)\right|$, and analogously for all other metrics including $g^{(i)}, i=1, \ldots, n$.

Further, a $\bar{g}^{(0)}$-covariant derivative of a given function $\alpha$ w.r.t. $x^{\mu}$ is denoted by $\alpha_{; \mu}$, its partial derivative also by $\alpha_{, \mu}$, and $(\partial \alpha)(\partial \beta):=\bar{g}^{(0) \mu \nu} \alpha_{, \mu} \beta_{, \nu}$.

On $\bar{M}_{0}$, the Laplace-Beltrami operator $\Delta\left[\bar{g}^{(0)}\right]=\frac{1}{\sqrt{\left|\bar{g}^{(0)}\right|}} \frac{\partial}{\partial x^{\mu}}\left(\sqrt{\left|\bar{g}^{(0)}\right|} \bar{g}^{(0) \mu \nu} \frac{\partial}{\partial x^{\nu}}\right)$, transforms under the conformal map $\bar{g}^{(0)} \mapsto e^{2 \bar{\gamma}} \bar{g}^{(0)}$ according to

$$
\begin{aligned}
\Delta\left[e^{2 \bar{\gamma}} \bar{g}^{(0)}\right] & =e^{-2 \bar{\gamma}} \Delta\left[\bar{g}^{(0)}\right]-e^{-2 \bar{\gamma}} \bar{g}^{(0)^{\mu \nu}}\left(\Gamma\left[e^{2 \bar{\gamma}} \bar{g}^{(0)}\right]-\Gamma\left[\bar{g}^{(0)}\right]\right)_{\mu \nu}^{\lambda} \frac{\partial}{\partial x^{\lambda}} \\
& =e^{-2 \bar{\gamma}}\left(\Delta\left[\bar{g}^{(0)}\right]+\left(D_{0}-2\right) g^{(0)^{\mu \nu}} \frac{\partial \bar{\gamma}}{\partial x^{\mu}} \frac{\partial}{\partial x^{\nu}}\right)
\end{aligned}
$$


where $\Gamma$ denotes the Levi-Civita connection.

The Levi-Civita connection $\Gamma$ corresponding to (2.6) does not decompose multidimensionally, and neither does the Riemann tensor. The latter is a section in $\mathfrak{T}_{3}^{1} M$ which is not given as a pullback to $\bar{M}_{0}$ of a section in the direct sum $\oplus_{i=1}^{n} \mathfrak{T}_{3}^{1} M_{i}$ of corresponding tensor bundles over the factor manifolds.

However, with (2.6) the Ricci tensor decomposes again multidimensionally:

$$
\operatorname{Ric}[g]=\operatorname{Ric}^{(0)}\left[g^{(0)}, \bar{\gamma} ; \phi\right] \oplus_{i=1}^{n} \operatorname{Ric}^{(i)}\left[g^{(0)}, \bar{\gamma} ; g^{(i)}, \phi\right],
$$

where

$$
\begin{aligned}
\operatorname{Ric}_{\mu \nu}^{(0)}:= & R_{\mu \nu}\left[g^{(0)}\right]+g_{\mu \nu}^{(0)}\left\{-\triangle\left[g^{(0)}\right] \bar{\gamma}+\left(2-D_{0}\right)(\partial \bar{\gamma})^{2}-\partial \bar{\gamma} \sum_{j=1}^{n} d_{j} \partial \phi^{j}\right\} \\
& +\left(2-D_{0}\right)\left(\bar{\gamma}_{; \mu \nu}-\bar{\gamma}_{, \mu} \bar{\gamma}_{, \nu}\right)-\sum_{i=1}^{n} d_{i}\left(\phi_{; \mu \nu}^{i}-\phi_{, \mu}^{i} \bar{\gamma}_{, \nu}-\phi_{, \nu}^{i} \bar{\gamma}_{, \mu}+\phi_{, \mu}^{i} \phi_{, \nu}^{i}\right), \\
\operatorname{Ric}_{m_{i} n_{i}}^{(i)}:= & R_{m_{i} n_{i}}\left[g^{(i)}\right]-e^{2 \phi^{i}-2 \bar{\gamma}^{(i)}} g_{m_{i} n_{i}}\left\{\triangle\left[g^{(0)}\right] \phi^{i}+\left(\partial \phi^{i}\right)\left[\left(D_{0}-2\right) \partial \bar{\gamma}+\sum_{j=1}^{n} d_{j} \partial \phi^{j}\right]\right\}, \\
& i=1, \ldots, n,
\end{aligned}
$$

The corresponding Ricci curvature scalar reads

$$
\begin{aligned}
R[g]= & e^{-2 \bar{\gamma}} R\left[\bar{g}^{(0)}\right]+\sum_{i=1}^{n} e^{-2 \beta^{i}} R\left[g^{(i)}\right]-e^{-2 \bar{\gamma}} \bar{g}^{(0) \mu \nu}\left(\left(D_{0}-2\right)\left(D_{0}-1\right) \frac{\partial \bar{\gamma}}{\partial x^{\mu}} \frac{\partial \bar{\gamma}}{\partial x^{\nu}}\right. \\
& \left.+\sum_{i, j=1}^{n}\left(d_{i} \delta_{i j}+d_{i} d_{j}\right) \frac{\partial \beta^{i}}{\partial x^{\mu}} \frac{\partial \beta^{j}}{\partial x^{\nu}}+2\left(D_{0}-2\right) \sum_{i=1}^{n} d_{i} \frac{\partial \bar{\gamma}}{\partial x^{\mu}} \frac{\partial \beta^{i}}{\partial x^{\nu}}\right) \\
& -2 e^{-2 \bar{\gamma}} \Delta\left[\bar{g}^{(0)}\right]\left(\left(D_{0}-1\right) \bar{\gamma}+\sum_{i=1}^{n} d_{i} \beta^{i}\right) .
\end{aligned}
$$

Let us now set

$$
f \equiv f[\bar{\gamma}, \beta]:=\left(D_{0}-2\right) \bar{\gamma}+\sum_{j=1}^{n} d_{j} \beta^{j},
$$

where $\beta$ is the vector field with the dilatonic scalar fields $\beta^{i}$ as components. (Note that $f$ can be resolved for $\bar{\gamma} \equiv \bar{\gamma}[f, \beta]$ if and only if $D_{0} \neq 2$. The singular case $D_{0}=2$ is discussed in $[10$.) Then, (2.14) can also be written as

$$
R[g]-e^{-2 \bar{\gamma}} R\left[\bar{g}^{(0)}\right]-\sum_{i=1}^{n} e^{-2 \beta^{i}} R_{i}=
$$




$$
\begin{gathered}
=-e^{-2 \bar{\gamma}}\left\{\sum_{i=1}^{n} d_{i}\left(\partial \beta^{i}\right)^{2}+(\partial f)^{2}+\left(D_{0}-2\right)(\partial \bar{\gamma})^{2}+2 \Delta\left[\bar{g}^{(0)}\right](f+\bar{\gamma})\right\} \\
=-e^{-2 \bar{\gamma}}\left\{\sum_{i=1}^{n} d_{i}\left(\partial \beta^{i}\right)^{2}+\left(D_{0}-2\right)(\partial \bar{\gamma})^{2}-(\partial f) \partial(f+2 \bar{\gamma})+R_{B}\right\}, \\
R_{B}:=\frac{1}{\sqrt{\left|\bar{g}^{(0)}\right|}} e^{-f} \partial_{\mu}\left[2 e^{f} \sqrt{\left.\left|\bar{g}^{(0)}\right| \bar{g}^{(0) \mu \nu} \partial_{\nu}(f+\bar{\gamma})\right],}\right.
\end{gathered}
$$

where the last term will yield just a boundary contribution (2.22) to the action (2.21) below.

Let us assume all $M_{i}, i=1, \ldots, n$, to be connected and oriented. The Riemann-Lebesgue volume form on $M_{i}$ is denoted by

$$
\tau_{i}:=\operatorname{vol}\left(g^{(i)}\right)=\sqrt{\left|g^{(i)}\left(y_{i}\right)\right|} d y_{i}^{1} \wedge \ldots \wedge d y_{i}^{d_{i}},
$$

and the total internal space volume by

$$
\mu:=\prod_{i=1}^{n} \mu_{i}, \quad \mu_{i}:=\int_{M_{i}} \tau_{i}=\int_{M_{i}} \operatorname{vol}\left(g^{(i)}\right) .
$$

If all of the spaces $M_{i}, i=1, \ldots, n$ are compact, then the volumes $\mu_{i}$ and $\mu$ are finite, and so are also the numbers $\rho_{i}=\int_{M_{i}} \operatorname{vol}\left(g^{(i)}\right) R\left[g^{(i)}\right]$. However, a non-compact $M_{i}$ might have infinite volume $\mu_{i}$ or infinite $\rho_{i}$. Nevertheless, by the $R$-homogeneity of $g^{(i)}$ (in particular satisfied for Einstein spaces), the ratios $\frac{\rho_{i}}{\mu_{i}}=R\left[g^{(i)}\right], i=1, \ldots, n$, are just finite constants. In any case, the $D$-dimensional coupling constant $\kappa$ can be tuned such that, under the dimensional reduction pr : $M \rightarrow \bar{M}_{0}$,

$$
\kappa_{0}:=\kappa \cdot \mu^{-\frac{1}{2}}
$$

becomes the $D_{0}$-dimensional physical coupling constant. If $D_{0}=4$, then $\kappa_{0}{ }^{2}=8 \pi G_{N}$, where $G_{N}$ is the Newton constant. The limit $\kappa \rightarrow \infty$ for $\mu \rightarrow \infty$ is in particular harmless, if $D$ dimensional gravity is given purely by curvature geometry, without additional matter fields. If however this geometry is coupled with finite strength to additional (matter) fields, one should indeed better take care to have all internal spaces $M_{i}, i=1, \ldots, n$ compact. Often this can be achieved by factorizing with an appropriate finite symmetry group.

With the total dimension $D, \kappa^{2}$ a $D$-dimensional gravitational constant we consider a purely gravitational action of the form

$$
S=\frac{1}{2 \kappa^{2}} \int_{N} d^{D} z \sqrt{|g|}\{R[g]\}+S_{\mathrm{GHY}} .
$$


Here a (generalized) Gibbons-Hawking-York [11, 12 type boundary contribution $S_{\mathrm{GHY}}$ to the action is taken to cancel boundary terms. Eqs.(2.16) and (2.17) show that $S_{\mathrm{GHY}}$ should be taken in the form

$$
\begin{aligned}
S_{\mathrm{GHY}} & :=\frac{1}{2 \kappa^{2}} \int_{N} d^{D} z \sqrt{|g|}\left\{e^{-2 \bar{\gamma}} R_{B}\right\} \\
& =\frac{1}{\kappa_{0}^{2}} \int_{\bar{M}_{0}} d^{D_{0}} x \frac{\partial}{\partial x^{\lambda}}\left(e^{f} \sqrt{\left|\bar{g}^{(0)}\right|} \bar{g}^{(0) \lambda \nu} \frac{\partial}{\partial x^{\nu}}(f+\bar{\gamma})\right),
\end{aligned}
$$

which is just a pure boundary term in form of an effective $D_{0}$-dimensional flow through $\partial \bar{M}_{0}$.

After dimensional reduction the action (2.21) reads

$$
\begin{array}{r}
S=\frac{1}{2 \kappa_{0}^{2}} \int_{\bar{M}_{0}} d^{D_{0}} x \sqrt{\left|\bar{g}^{(0)}\right|} e^{f}\left\{R\left[\bar{g}^{(0)}\right]+(\partial f)(\partial[f+2 \bar{\gamma}])-\sum_{i=1}^{n} d_{i}\left(\partial \beta^{i}\right)^{2}\right. \\
\left.-\left(D_{0}-2\right)(\partial \bar{\gamma})^{2}+e^{2 \bar{\gamma}}\left[\sum_{i=1}^{n} e^{-2 \beta^{i}} R_{i}\right]\right\},
\end{array}
$$

where $e^{f}$ is a dilatonic scalar field coupling to the $D_{0}$-dimensional geometry on $\bar{M}_{0}$.

According to the considerations above, due to the conformal reparametrization invariance of the geometry on $\bar{M}_{0}$, we should fix a conformal frame on $\bar{M}_{0}$. But then in $(2.23) \bar{\gamma}$, and with (2.15) also $f$, is no longer independent from the vector field $\beta$, but rather

$$
\bar{\gamma} \equiv \bar{\gamma}[\beta], \quad f \equiv f[\beta]
$$

Then, modulo the conformal factor $e^{f}$, the dilatonic kinetic term of (2.23) takes the form

$$
(\partial f)(\partial[f+2 \bar{\gamma}])-\sum_{i=1}^{n} d_{i}\left(\partial \beta^{i}\right)^{2}-\left(D_{0}-2\right)(\partial \bar{\gamma})^{2}=-G_{i j}\left(\partial \beta^{i}\right)\left(\partial \beta^{j}\right),
$$

with $G_{i j} \equiv{ }^{(\bar{\gamma})} G_{i j}$, where

$$
\begin{aligned}
&{ }^{(\bar{\gamma})} G_{i j}:={ }^{(\mathrm{BD})} G_{i j}-\left(D_{0}-2\right)\left(D_{0}-1\right) \frac{\partial \bar{\gamma}}{\partial \beta^{i}} \frac{\partial \bar{\gamma}}{\partial \beta^{j}}-2\left(D_{0}-1\right) d_{(i} \frac{\partial \bar{\gamma}}{\partial \beta^{j)}}, \\
&{ }^{(\mathrm{BD})} G_{i j}:=\delta_{i j} d_{i}-d_{i} d_{j} .
\end{aligned}
$$

For $D_{0} \neq 2$, we can write equivalently $G_{i j} \equiv{ }^{(f)} G_{i j}$, where

$$
\begin{array}{r}
{ }^{(f)} G_{i j}:={ }^{(\mathrm{E})} G_{i j}-\frac{D_{0}-1}{D_{0}-2} \frac{\partial f}{\partial \beta^{i}} \frac{\partial f}{\partial \beta^{j}}, \\
{ }^{(\mathrm{E})} G_{i j}:=\delta_{i j} d_{i}+\frac{d_{i} d_{j}}{D_{0}-2} .
\end{array}
$$


For $D_{0}=1, G_{i j}={ }^{(\mathrm{E})} G_{i j}={ }^{(\mathrm{BD})} G_{i j}$ is independent of $\bar{\gamma}$ and $f$. Note that the metrics (2.27) and $(2.29)$ (with $D_{0} \neq 2$ ) may be diagonalized to $\left(\mp( \pm)^{\delta_{1 D_{0}}}\right)^{\delta_{1 i}} \delta_{i j}$ respectively, by homogeneous linear minisuperspace coordinate transformations $\beta{ }^{T} \rightarrow z$ and $\beta \stackrel{Q}{\mapsto} \varphi$, explicitly given by components

$$
\begin{aligned}
& z^{1}:={ }^{(\mathrm{BD})} q^{-1} \sum_{j=1}^{n} d_{j} \beta^{j}, \varphi^{1}:={ }^{(\mathrm{E})} q^{-1} \sum_{j=1}^{n} d_{j} \beta^{j} \\
& z^{i} \equiv \varphi^{i} \quad:=\quad\left[d_{i-1} / \Sigma_{i-1} \Sigma_{i}\right]^{1 / 2} \sum_{j=i}^{n} d_{j}\left(\beta^{j}-\beta^{i-1}\right)
\end{aligned}
$$

$i=2, \ldots, n$, where with $D^{\prime}:=D-D_{0}$ and $\Sigma_{k}:=\sum_{i=k}^{n} d_{i}$,

$$
{ }^{(\mathrm{BD})} q:=\sqrt{\frac{D^{\prime}}{D^{\prime}-1}}, \quad{ }^{(\mathrm{E})} q:=\sqrt{\frac{D^{\prime}\left(D_{0}-2\right)}{D^{\prime}+D_{0}-2}} .
$$

So, after fixing a conformal reparametrization gauge for the geometry on $M_{0}$, (2.21) becomes a $\sigma$-model, where the vector field $\beta$ (or $z$ resp. $\varphi$ ) defines the coordinates of its $n$-dimensional target space. In the following, we will simplify notation by a summation convention for tensors over target space.

In general, for $n>2$ and non-constant functional $\gamma[\beta]$, the minisuperspace metric given by (2.25) and the conformally related target space metric may not even be conformally flat. However, for constant $\bar{\gamma}$, 2.26) reduces to (2.27), whence target space is conformally flat, namely it is related to $n$-dimensional Minkowski space by a conformal scale factor

$$
\varphi \equiv \varphi(\beta):=\prod_{l=1}^{n} e^{d_{l} \beta^{l}}=e^{(\mathrm{BD})} q z^{1}=e^{(\mathrm{E}) q \varphi^{1}},
$$

which is proportional to the total internal space volume.

In the case $D_{0} \neq 2$, for non-constant functional $f[\beta]$, the target space may again in general not be conformally flat for $n>2$. However, for constant $f$, (2.28) reduces to (2.29), whence, target space is a flat $n$-dimensional space, namely an Euclidean one for $D_{0}>2$, and a Minkowskian one for $D_{0}=1$.

After gauging $\bar{\gamma}$, setting $m:=\kappa_{0}^{-2}$, (2.23) yields a $\sigma$-model in the form

$$
\begin{aligned}
& { }^{(\bar{\gamma})} S=\left.\int_{\bar{M}_{0}} d^{D_{0}} x \sqrt{\left|\bar{g}^{(0)}\right|}\right|^{(\bar{\gamma})} N^{D_{0}} \quad \varphi(\beta)\left\{\frac{m}{2}(\bar{\gamma}) N^{-2}\left[R\left[\bar{g}^{(0)}\right]-{ }^{(\bar{\gamma})} G_{i j}\left(\partial \beta^{i}\right)\left(\partial \beta^{j}\right)\right]\right. \\
& \left.-{ }^{(\mathrm{BD})} V(\beta)\right\} \text {, }
\end{aligned}
$$




$$
\text { where } \quad \begin{aligned}
{ }^{(\mathrm{BD})} V(\beta) & :=m\left[-\frac{1}{2} \sum_{i=1}^{n} R\left[g^{(i)}\right] e^{-2 \beta^{i}}\right], \\
{ }^{(\bar{\gamma})} N & :=e^{\bar{\gamma}} .
\end{aligned}
$$

Note that, the potential (2.34) and the conformal factor $\phi(\beta):=\prod_{i=1}^{n} e^{d_{i} \beta^{i}}$ are gauge invariant. Analogously, the $\sigma$-model action from (2.23) gauging $f$ can also be written as

$$
\begin{aligned}
{ }^{(f)} S=\int_{\bar{M}_{0}} d^{D_{0}} x \sqrt{\mid \bar{g}^{(0)}{ }^{(f)} N^{D_{0}}} \quad\left\{\frac{m}{2}(f) N^{-2}\left[R\left[\bar{g}^{(0)}\right]-{ }^{(f)} G_{i j}\left(\partial \beta^{i}\right)\left(\partial \beta^{j}\right)\right]\right. \\
\left.-{ }^{(\mathrm{E})} V(\beta)\right\} \\
{ }^{(\mathrm{E})} V(\beta):=m \Omega^{2}\left[-\frac{1}{2} \sum_{i=1}^{n} R\left[g^{(i)}\right] e^{\left.-2 \beta^{i}\right]}\right. \\
(f) N:=e^{\frac{f}{D_{0}-2}}
\end{aligned}
$$

where the function $\Omega$ on $\bar{M}_{0}$ is defined as

$$
\Omega:=\varphi^{\frac{1}{2-D_{0}}} .
$$

Note that, with $\Omega$ also the potential (2.37) is gauge invariant, and the dilatonic target-space, though not even conformally flat in general, is flat for constant $f$.

In fact, Eqs. (2.33)-(2.35) and (2.36)-(2.38) show that there are at least two special frames.

The first one corresponds to the gauge $\bar{\gamma} \stackrel{!}{=} 0$. In this case ${ }^{(\bar{\gamma})} N=1$, the minisuperspace metric (2.26) reduces to the Minkowskian (2.27), the dilatonic scalar field becomes proportional to the internal space volume, $e^{f[\beta]}=\varphi(\beta)=\prod_{i=1}^{n} e^{d_{i} \beta^{i}}$, and (2.33) describes a generalized $\sigma$-model with conformally Minkowskian target space. The Minkowskian signature implies a negative sign in the dilatonic kinetic term. This frame is usually called the Brans-Dicke one, because $\varphi=e^{f}$ here plays the role of a Brans-Dicke scalar field.

The second distinguished frame corresponds to the gauge $f \stackrel{!}{=} 0$, where $\bar{\gamma}=\frac{1}{2-D_{0}} \sum_{i=1}^{n} d_{i} \beta^{i}$ is well-defined only for $D_{0} \neq 2$. In this case ${ }^{(f)} N=1$, the minisuperspace metric (2.28) reduces to the Euclidean (2.29), and (2.36) describes a self-gravitating $\sigma$-model with Euclidean target space. Hence all dilatonic kinetic terms have positive signs. This frame is usually called the Einstein one, because it describes an effective $D_{0}$-dimensional Einstein theory with additional minimally coupled scalar fields. For multidimensional geometries with $D_{0}=2$ the Einstein frame fails to exist, which reflects the well-known fact that two-dimensional Einstein equations are trivially satisfied without implying any dynamics.

For $D_{0}=1$, the action of both (2.33) and (2.36) was shown in [13] (and previously in [14, 15]) to take the form of a classical particle motion on minisuperspace, whence different 
frames correspond are just related by a time reparametrization. More generally, for $D_{0} \neq 2$ and $\left(\bar{M}_{0}, \bar{g}(0)\right)$ a vacuum space-time, the $\sigma$-model (2.36) with the gauge $f \stackrel{!}{=} 0$ describes the dynamics of a massive $\left(D_{0}-1\right)$-brane within a potential (2.37) on its target minisuperspace.

In fact, the target space is in general a conformally homogeneous space, and in the Einstein frame a homogeneous one. Once its isometry group $\mathfrak{G}$ and isotropy group $\mathfrak{H}$ are known, it is clear that the sigma model (2.36) can also be written in matrix form

$$
\begin{gathered}
{ }^{(f)} S=\int_{\bar{M}_{0}} d^{D_{0}} x \sqrt{\left|\bar{g}^{(0)}\right|} N^{D_{0}}(\mathcal{M}) \quad\left\{\frac{m}{2} N^{-2}(\mathcal{M})\left[R\left[\bar{g}^{(0)}\right]+g^{(0) \mu \nu} B \operatorname{Tr}_{\rho}\left(\partial_{\mu} \mathcal{M} \partial_{\nu} \mathcal{M}^{-1}\right)\right]\right. \\
\left.-{ }^{(\mathrm{E})} U(\mathcal{M})\right\}
\end{gathered}
$$

with $\mathcal{M} \in \rho(G)$ where $\rho$ is an appropriate coset representation of the target space $\mathfrak{M}:=\mathfrak{G} / \mathfrak{H}$, ${ }^{(\mathrm{E})} U$ is now the corresponding potential on $\mathfrak{M}, N$ a gauge function on $\mathfrak{M}$, and $B$ a normalization.

For $D_{0}=4$, eq. (2.40) can also be written in the Einstein frame as

$$
\begin{gathered}
{ }^{(E)} S=\int_{\bar{M}_{0}} \quad\left\{\frac{m}{2}\left[\operatorname{Tr} \Omega \wedge * \Sigma+B \operatorname{Tr}{ }_{\rho} d \mathcal{M} \wedge * d \mathcal{M}^{-1}\right]\right. \\
\left.-{ }^{(\mathrm{E})} U(\mathcal{M}) * 1\right\}
\end{gathered}
$$

where $\Omega$ is the curvature 2 -form, $\Sigma:=e \wedge e$ and $\bar{g}^{(0)}$ are given by the $D_{0}$-dimensional soldering 1-form $e$, and the Hodge star is taken w.r.t. $\left(\bar{M}, g^{(0)}\right)$. The form (2.41) is a then a convenient starting point for the canonical quantization procedure.

In the purely gravitational model consider so far $\mathfrak{M}$ is a finite dimensional and homogeneous with a transitive Abelian group. In the following section let us add minimally coupled scalar and $p+2$-form matter fields and investigate the extension of the resulting target space $\mathfrak{M}$.

\section{III. $\sigma$-model with minimally coupled scalars and $p+2-$ forms}

We now couple the purely gravitational action (2.21) to additional matter fields of scalar and generalized Maxwell type, i.e. we consider now the action

$$
\begin{aligned}
2 \kappa^{2}\left[S\left[g, \phi, F^{a}\right]-S_{\mathrm{GHY}}\right]=\int_{N} d^{D} z \sqrt{|g|} & \left\{R[g]-C_{\alpha \beta} g^{M N} \partial_{M} \Phi^{\alpha} \partial_{N} \Phi^{\beta}\right. \\
& \left.-\sum_{a \in \Delta} \frac{\eta_{a}}{n_{a} !} \exp \left[2 \lambda_{a}(\Phi)\right]\left(F^{a}\right)^{2}\right\}
\end{aligned}
$$


of a self-gravitating $\sigma$ model on $M$ with topological term $S_{\mathrm{GHY}}$. Here the $l$-dimensional target space, defined by a vector field $\phi$ with scalar components $\phi^{\alpha}, \alpha=1, \ldots, l$, is coupled to several antisymmetric $n_{a}$-form fields $F^{a}$ via 1 -forms $\lambda_{a}, a \in \Delta$. For consistency, we have to demand of course that all fields are internally homogeneous. We will see below how this gives rise to an effective $l+|\Delta|$-dimensional target-space extension. Note also that for convenience here we work with fields $\phi$ and $F$ which differ from the actual (physical) matter fields by a rescaling with the square root of the coupling constant.

With $I \subset\{1, \ldots, n\}$, the generalized Maxwell fields $F^{a}$ are located on $\left(n_{a}-1\right)$-dimensional world sheets

$$
\begin{gathered}
M_{I}:=\prod_{i \in I} M_{i}=M_{i_{1}} \times \ldots \times M_{i_{k}}, \\
n_{a}-1=D(I):=\sum_{i \in I} d_{i}=d_{i_{1}}+\ldots+d_{i_{k}} .
\end{gathered}
$$

of different $\left(n_{a}-2\right)$-branes, labeled for each $a$ by the sets $I$ in a certain subset $\Omega_{a}$ of the power set of $\{1, \ldots, n\}$. Variation of (3.1) yields the field equations

$$
\begin{aligned}
R_{M N}-\frac{1}{2} g_{M N} R & =T_{M N}, \\
C_{\alpha \beta} \Delta[g] \phi^{\beta}-\sum_{a \in \Delta} \frac{\eta_{a} \lambda_{a}^{\alpha}}{n_{a} !} e^{2 \lambda_{a}(\phi)}\left(F^{a}\right)^{2} & =0, \\
\nabla_{M_{1}}[g]\left(e^{2 \lambda_{a}(\phi)} F^{a, M_{1} M_{2} \ldots M_{n_{a}}}\right) & =0,
\end{aligned}
$$

$a \in \Delta, \alpha=1, \ldots, l$.

In (3.4) the $D$-dimensional energy-momentum resulting from (3.1) is given by a sum

$$
T_{M N}:=\sum_{\alpha=1}^{l} T_{M N}\left[\phi^{\alpha}, g\right]+\eta_{a} \sum_{a \in \Delta} e^{2 \lambda_{a}(\phi)} T_{M N}\left[F^{a}, g\right],
$$

of contributions from scalar and generalized Maxwell fields,

$$
\begin{aligned}
T_{M N}\left[\phi^{\alpha}, g\right] & :=C_{\alpha \beta} \partial_{M} \phi^{\alpha} \partial_{N} \phi^{\alpha}-\frac{1}{2} g_{M N} \partial_{P} \phi^{\alpha} \partial^{P} \phi^{\alpha}, \\
T_{M N}\left[F^{a}, g\right] & :=\frac{1}{n_{a} !}\left[-\frac{1}{2} g_{M N}\left(F^{a}\right)^{2}+n_{a} F_{M M_{2} \ldots M_{n_{a}}}^{a} F_{N}^{a M_{2} \ldots M_{n_{a}}}\right] .
\end{aligned}
$$

We give now a sufficient criterion for the energy-momentum tensor (3.7) to decompose multidimensionally. 
Let $W_{1}:=\left\{i \mid i>0, d_{i}=1\right\}$ be the label set of 1-dimensional factor spaces of the multidimensional decomposition, and set $n_{1}:=\left|W_{1}\right|$. Define

$$
W(a ; i, j):=\left\{(I, J) \mid I, J \in \Omega_{a},(I \cap J) \cup\{i\}=I \not \supset j,(I \cap J) \cup\{j\}=J \not \supset i\right\}
$$

Then the following holds.

Theorem: If for $n_{1}>1$ the $p$-branes satisfy the condition for all $a \in \Delta, i, j \in W_{1}$ with $i \neq j$, the condition

$$
W(a ; i, j) \stackrel{!}{=} \emptyset \quad \forall a \in \Delta \forall i, j \in W_{1}
$$

then the energy-momentum (3.7) decomposes multidimensionally without further constraints.

Proof: The only possible obstruction to the multidimensional decomposition of (3.7) comes from the second term of (3.9), $F^{a}{ }_{M M_{2} \ldots M_{n_{a}}} F_{N}^{a}{ }_{N} \ldots M_{n_{a}}$ when the indices $M$ and $N$ take values in different index sets labeling different 1-dimensional factor spaces. The theorem then follows just from the antisymmetry of the $F$-fields.

Corollary: A sufficient condition for the multidimensional decomposition of (3.7) is

$$
n_{1} \stackrel{!}{\leq} 1
$$

If condition (3.11) does not hold, multidimensional decomposability of (3.7) may impose additional non-trivial constraints on the $p+2$-form fields.

Let us now specify the components of the $F$-fields of generalized electric and magnetic type.

Antisymmetric fields of generalized electric type, are given by scalar potential fields $\Phi^{a, I}$, $a \in \Delta, I \in \Omega_{a}$, which compose to a $\left(\sum_{a \in \Delta}\left|\Omega_{a}\right|\right)$-dimensional vector field $\Phi$. Magnetic type fields are just given as the duals of appropriate electric ones.

$$
\begin{aligned}
F^{e, I} & =d \Phi^{e, I} \wedge \tau(I) \\
F^{m, I} & =e^{-2 \lambda_{a}(\phi)} *\left(d \Phi^{m, I} \wedge \tau(J)\right) .
\end{aligned}
$$

In the Einstein frame, the action then reduces to

$$
\begin{gathered}
{ }^{(\mathrm{E})} S\left[g^{(0)}, \beta, \phi, \Phi\right]=\int_{M_{0}} d^{D_{0}} x \sqrt{\left|g^{(0)}\right|}\left\{\frac { m } { 2 } \left[R\left[g^{(0)}\right]-G_{i j}\left(\partial \beta^{i}\right)\left(\partial \beta^{j}\right)\right.\right. \\
\left.\left.-C_{\alpha \beta}\left(\partial \phi^{\alpha}\right)\left(\partial \phi^{\beta}\right)-\sum_{a \in \Delta, I \in \Omega_{a}} \varepsilon_{a, I} e^{2\left(\lambda_{a}(\phi)-d_{i} \beta^{i}\right)}\left(\partial \Phi^{a, I}\right)^{2}\right]-{ }^{(\mathrm{E})} V(\beta)\right\},
\end{gathered}
$$

which corresponds to an purely Einsteinian $\sigma$-model on $M_{0}$ with extended $\left(n+l+\sum_{a \in \Delta}\left|\Omega_{a}\right|\right)$ dimensional target space and dilatonic potential (2.37). Here and below we will consider by 
default the Einstein frame, and set correspondingly $G_{i j}:={ }^{(\mathrm{E})} G_{i j}$. In (3.15) and below a summation convention is assumed also on the extended target space.

For convenience, let us introduce the topological numbers

$$
l_{j I}:=-\sum_{i \in I} D_{i} \delta_{j}^{i}, \quad j=1, \ldots, n
$$

and with $N:=n+l$ define and define a $N \times|S|$-matrix

$$
L=\left(L_{A s}\right)=\left(\begin{array}{c}
L_{i s} \\
L_{\alpha s}
\end{array}\right):=\left(\begin{array}{c}
l_{i I} \\
\lambda_{\alpha a}
\end{array}\right)
$$

a $N$-dimensional vector field $\left(\sigma^{A}\right):=\left(\beta^{i}, \phi^{\alpha}\right), A=1, \ldots, n, n+1, \ldots, N$, composed by dilatonic and matter scalar fields, and a non-degenerate (block-diagonal) $N \times N$-matrix

$$
\hat{G}=\left(\hat{G}_{A B}\right)=\left(\begin{array}{cc}
G_{i j} & 0 \\
0 & C_{\alpha \beta}
\end{array}\right)
$$

With these definitions, (3.15) takes the form

$$
S_{0}=\int_{M_{0}} d^{D_{0}} x \sqrt{\left|g^{(0)}\right|}\left\{\frac{m}{2}\left[R\left[g^{(0)}\right]-\hat{G}_{A B} \partial \sigma^{A} \partial \sigma^{B}-\sum_{s \in S} \varepsilon_{s} e^{2 L_{A s} \sigma^{A}}\left(\partial \Phi^{s}\right)^{2}\right]-{ }^{(\mathrm{E})} V(\sigma)\right\}
$$

\section{Solution with Abelian target-space}

In the this section we consider the $\sigma$-model (3.15) without the $\Phi$ fields from the $p+2$-forms, whence the target-space is the $n+l$-dimensional Abelian one, and present a particularly interesting vacuum solution.

We derive for $D_{0} \neq 2$ an new exact Ricci flat multidimensional solution for the effective $\sigma$-model (3.15) in the harmonic gauge $\left(2-D_{0}\right) \bar{\gamma} \stackrel{!}{=} d_{i} \beta^{i}$ with zero potential (2.37) and zero $\Phi$. The field equation then read

$$
\begin{aligned}
G_{i j} \partial_{\mu} \beta^{i} \partial_{\nu} \beta^{j}+C_{\alpha \beta} \partial_{\mu} \phi^{\alpha} \partial_{\nu} \phi^{\beta} & =0, & & \mu, \nu=0, \ldots, D_{0}-1, \\
{ }^{(\mathrm{E})} G_{i j} \Delta\left[\bar{g}^{(0)}\right] \beta^{j} & =0, & & i=1, \ldots, n, \\
C_{\alpha \beta} \Delta\left[\bar{g}^{(0)}\right] \phi^{\beta} & =0, & & \alpha=1, \ldots, l .
\end{aligned}
$$

In particular, we now solve these equations with flat $\left(\bar{M}_{0}, \bar{g}^{(0)}\right)$. In this case, there exist $g$ harmonic $\bar{M}_{0^{-c o o r d i n a t e s}} \tau^{\mu}, \mu=0, \ldots, D_{0}-1$. Let $g^{(0)}=e^{-2 \bar{\gamma}} \eta_{\mu \nu} d \tau^{\mu} d \tau^{\nu}$. In such harmonic 
coordinates, equations (4.2) and (4.3) are solved by

$$
\begin{aligned}
\beta^{i}=b_{\mu}^{i} \tau^{\mu}+c^{i}, & i=1, \ldots, n, \\
\phi^{\alpha}=b_{\mu}^{n+\alpha} \tau^{\mu}+c^{n+\alpha}, & \alpha=1, \ldots, l .
\end{aligned}
$$

We set

$$
\varphi_{\mu}^{i}:=\frac{\partial}{\partial \tau^{\mu}} \varphi^{i}, \quad \mu=0, \ldots, D_{0}-1 .
$$

With (4.4), the harmonic gauge condition reads

$$
A_{\mu}:={ }^{(\mathrm{E})} q \varphi_{\mu}^{1}=\sum_{i} d_{i} b_{\mu}^{i} \stackrel{!}{=} 0, \quad \quad \mu=0, \ldots, D_{0}-1
$$

With the harmonic gauge constraint (4.7), Eq. (4.1) then reads

$$
\begin{gathered}
\sum_{i=2}^{n} \varphi_{\mu}^{i} \varphi_{\nu}^{i}+\sum_{\alpha \beta=1}^{l} C_{\alpha \beta} b_{\mu}^{\alpha} b_{\nu}^{\beta}=\sum_{i=1}^{n} d_{i} b_{\mu}^{i} b_{\nu}^{i}+\sum_{\alpha \beta=1}^{l} C_{\alpha \beta} b_{\mu}^{\alpha} b_{\nu}^{\beta} \stackrel{!}{=} 0 \\
\mu, \nu=0, \ldots, D_{0}-1
\end{gathered}
$$

For convenience, one can set $c^{A}:=0, A=1, \ldots, n+l$. Then $\gamma=0$, whence the harmonic coordinates are simultaneously proper coordinates, and the solution reads explicitly,

$$
g=\eta_{\mu \nu} d \tau^{\mu} \otimes d \tau^{\nu}+\sum_{i=1}^{n} e^{2 b_{\lambda}^{i} \tau^{\lambda}} g^{(i)}
$$

with linear coefficients $b_{\mu}^{i}, i=1, \ldots, n, \mu=0, \ldots, D_{0}-1$, satisfying $D_{0}$ linear constraints (4.7) (the harmonic gauge) and $D_{0}{ }^{2}$ quadratic constraints (4.8) (the harmonic Wheeler-de Witt constraints).

This solution shows a generalized inflationary behaviour, which extends the familiar notion of inflation w.r.t. time, as in cosmology, to inflation w.r.t. the internal degrees of freedom on the $D_{0}$-dimensional world manifold of an extended object. The constraint (4.7) implies that the total $\left(D-D_{0}\right)$ - dimensional volume remains constant (like in a steady state universe [16]) on the world manifold $M_{0}$, although here (unlike the stationary case [16]) individual factor spaces may undergo inflationary expansion or contraction in particular directions on $M_{0}$. In the standard cosmological case $D_{0}=1$, this solution agrees with the one described in [17]. 


\section{Orthobrane solutions with ${ }^{(\mathrm{E})} V=0$}

Now we present a class of solutions with ${ }^{(\mathrm{E})} V=0$, where the field equations read

$$
\begin{aligned}
R_{\mu \nu}\left[g^{(0)}\right]=\hat{G}_{A B} \partial_{\mu} \sigma^{A} \partial_{\nu} \sigma^{B}+\sum_{s \in S} \varepsilon_{s} e^{2 L_{A s} \sigma^{A}} \partial_{\mu} \Phi^{s} \partial_{\nu} \Phi^{s}, & \mu, \nu=1, \ldots, D_{0}, \\
\hat{G}_{A B} \triangle\left[g^{(0)}\right] \sigma^{B}-\sum_{s \in S} \varepsilon_{s} L_{A s} e^{2 L_{C s} \sigma^{C}}\left(\partial \Phi^{s}\right)^{2}=0, & A=1, \ldots, N, \\
\partial_{\mu}\left(\sqrt{\left|g^{(0)}\right|} g^{(0) \mu \nu} e^{2 L_{A s} \sigma^{A}} \partial_{\nu} \Phi^{s}\right)=0, & s \in S .
\end{aligned}
$$

For the Abelian part of the target space metric we set $\left(\hat{G}^{A B}\right):=\left(\hat{G}_{A B}\right)^{-1}$.

$$
<X, Y>:=X_{A} \hat{G}^{A B} X_{B}
$$

For $s \in S$ let us now consider vectors

$$
L_{s}=\left(L_{A s}\right) \in \mathbb{R}^{N}
$$

Definition: A non-empty set $S$ is called an orthobrane index set, iff there exists a family of real non-zero coefficients $\left\{\nu_{s}\right\}_{s \in S}$, such that

$$
<L_{s}, L_{r}>=\left(L^{T} \hat{G}^{-1} L\right)_{s r}=-\varepsilon_{s}\left(\nu_{s}\right)^{-2} \delta_{s r}, \quad s, r \in S .
$$

For $s \in S$ and $A=1, \ldots, N$, we set

$$
\alpha_{s}^{A}:=-\varepsilon_{s}\left(\nu_{s}\right)^{2} \hat{G}^{A B} L_{B s}
$$

Here, (5.6) is just an orthogonality condition for the vectors $L_{s}, s \in S$. Note that $\left\langle L_{s}, L_{s}\right\rangle$ has just the opposite sign of $\varepsilon_{s}, s \in S$. With the definition above, we obtain an existence criterion for solutions.

Theorem: Let $S$ be an orthobrane index set with coefficients (5.7). If for any $s \in S$ there is a function $H_{s}>0$ on $M_{0}$ such that

$$
\triangle\left[g^{(0)}\right] H_{s}=0
$$

i.e. $H_{s}$ is harmonic on $M_{0}$, then, the field configuration

$$
\begin{aligned}
R_{\mu \nu}\left[g^{(0)}\right]=0, & \mu, \nu=1, \ldots, D_{0}, \\
\sigma^{A}=\sum_{s \in S} \alpha_{s}^{A} \ln H_{s}, & A=1, \ldots, N, \\
\Phi^{s}=\frac{\nu_{s}}{H_{s}}, & s \in S,
\end{aligned}
$$


satisfies the field equations (5.1)-(5.3).

This theorem follows just from substitution of (5.6)-(5.11) into the equations of motion (5.1)(5.3). From (3.18), (3.17) and (5.4) we get

$$
<L_{s}, L_{r}>=G^{i j} l_{i I} l_{j J}+C^{\alpha \beta} \lambda_{\alpha a} \lambda_{\beta b},
$$

with $s=(a, I)$ and $r=(b, J)$ in $S\left(a, b \in \Delta, I \in \Omega_{a}, J \in \Omega_{b}\right)$. Here, the inverse of the dilatonic midisuperspace metric $G_{i j}$ is given by

$$
G^{i j}=\frac{\delta_{i j}}{D_{i}}+\frac{1}{2-D}
$$

whence, for $I, J \in \Omega$, with topological numbers $l_{i I}$ from (3.16), we obtain

$$
G^{i j} l_{i I} l_{j J}=D(I \cap J)+\frac{D(I) D(J)}{2-D},
$$

which is again a purely topological number.

We set $\nu_{a, I}:=\nu_{(a, I)}$. Then, due to (5.12) and (5.14), the orthobrane condition (5.6) reads

$$
D(I \cap J)+\frac{D(I) D(J)}{2-D}+C^{\alpha \beta} \lambda_{\alpha a} \lambda_{\beta b}=-\varepsilon(I)\left(\nu_{a, I}\right)^{-2} \delta_{a b} \delta_{I, J},
$$

for $a, b \in \Delta, I \in \Omega_{a}, I \in \Omega_{b}$. With $(a, I)=s \in S$, the coefficients (5.7) are

$$
\begin{aligned}
\alpha_{s}^{i} & =-\varepsilon(I) G^{i j} l_{j I} \nu_{a, I}^{2}=\varepsilon(I)\left(\sum_{j \in I} \delta_{j}^{i}+\frac{D(I)}{2-D}\right) \nu_{a, I}^{2}, \quad i=1, \ldots, n, \\
\alpha_{s}^{\beta} & =-\varepsilon(I) C^{\beta \gamma} \lambda_{\gamma a} \nu_{a, I}^{2}, \quad \beta=1, \ldots, l
\end{aligned}
$$

With $\left(\sigma^{A}\right)=\left(\phi^{i}, \varphi^{\beta}\right)$, according to (5.10),

$$
\begin{aligned}
\beta^{i} & =\sum_{s \in S} \alpha_{s}^{i} \ln H_{s}, \quad i=1, \ldots, n, \\
\phi^{\beta} & =\sum_{s \in S} \alpha_{s}^{\beta} \ln H_{s}, \quad \beta=1, \ldots, l,
\end{aligned}
$$

and the harmonic gauge reads

$$
\gamma=\sum_{s \in S} \alpha_{s}^{0} \ln H_{s}
$$


where

$$
\alpha_{s}^{0}:=\varepsilon(I) \frac{D(I)}{2-D} \nu_{a, I}^{2} .
$$

With $H_{a, I}:=H_{(a, I)},(5.16)$, (5.17), and (5.21), the solution of (5.9) - (5.11) reads

$$
\begin{aligned}
& g=\left(\prod_{s \in S} H_{s}^{2 \alpha_{s}^{0}}\right) g^{(0)}+\sum_{i=1}^{n}\left(\prod_{s \in S} H_{s}^{2 \alpha_{s}^{i}}\right) g^{(i)} \\
&=\left(\prod_{(a, I) \in S} H_{a, I}^{\varepsilon(I) 2 D(I) \nu_{a, I}^{2}}\right)^{1 /(2-D)}\left\{g^{(0)}+\sum_{i=1}^{n}\left(\prod_{(a, I) \in S, I \ni i} H_{a, I}^{\varepsilon(I) 2 \nu_{a, I}^{2}}\right) g^{(i)}\right\}, \\
& \text { with } \operatorname{Ric}\left[g^{(0)}\right]=0, \quad \operatorname{Ric}\left[g^{(i)}\right]=0, \quad i=1, \ldots n, \\
& \phi^{\beta}= \sum_{s \in S} \alpha_{s}^{\beta} \ln H_{s}=-\sum_{(a, I) \in S} \varepsilon(I) C^{\beta \gamma} \lambda_{\gamma a} \nu_{a, I}^{2} \ln H_{a, I}, \quad \beta=1, \ldots, l, \\
& A^{a}=\sum_{I \in \Omega_{a}} \frac{\nu_{a, I}}{H_{a, I}} \tau_{I}, \quad a \in \Delta,
\end{aligned}
$$

where forms $\tau_{I}$ are defined in (2.18), parameters $\nu_{s} \neq 0$ and $\lambda_{a}$ satisfy the orthobrane condition (5.15), $H_{s}$ are positive harmonic functions on $M_{0}$, and $\operatorname{Ric}\left[g^{(i)}\right]$ denotes the Ricci-tensor of $g^{(i)}$. Finally recall that these solutions are subject to the orthobrane constraints

$$
D(I \cap J)+\frac{D(I) D(J)}{2-D}+C^{\alpha \beta} \lambda_{\alpha a} \lambda_{\beta b}=-\varepsilon(I)\left(\nu_{a, I}\right)^{-2} \delta_{a b} \delta_{I, J}, \quad 0 \neq \nu_{a, I} \in \mathbb{R},
$$

for $a, b \in \Delta, I \in \Omega_{a}, I \in \Omega_{b}$. These condition lead to specific intersection rules for the $p$-branes involved. Some concrete examples of orthobrane solutions have been elaborated in [18].

For positive definite $\left(C_{\alpha \beta}\right)$ (or $\left(C^{\alpha \beta}\right)$ ) and $D_{0} \geq 2$, (5.25) implies

$$
\varepsilon(I)=-1,
$$

for all $I \in \Omega_{a}, a \in \Delta$. Then, the restriction $g_{\mid M_{I}}$ of the metric (5.22) to a membrane manifold $M_{I}$ has an odd number of negative eigenvalues, i.e. linearly independent time-like directions. However, if the metric $\left(C_{\alpha \beta}\right)$ in the space of scalar fields is not positive definite, then (5.26) may be violated for sufficiently negative $C^{\alpha \beta} \lambda_{\alpha a} \lambda_{\beta b}<0$.

\section{Target space structure}

Theorem: The target space $(\mathfrak{M}, \mathfrak{g})$ is a homogeneous space. 
Proof: The Killing vectors of a transitive subgroup of $\operatorname{Isom}(\mathfrak{M})$ can be determined explicitly.

$$
\begin{aligned}
V_{s} & :=\frac{\partial}{\partial \Phi^{s}}, \quad s \in S \\
U_{A} & :=\frac{\partial}{\partial x^{A}}-\sum_{s \in S} U_{A}^{s} \Phi^{s} \frac{\partial}{\partial \Phi^{s}}, \quad A=1, \ldots, N .
\end{aligned}
$$

Moreover, the Lie-algebra of the transitive group of isometries generated by (6.1) reads

$$
\begin{aligned}
{[U, U]=[V, V] } & =0 \\
{\left[U_{A}, V_{s}\right] } & =L_{A}^{s} V_{s}, \quad A=1, \ldots, N, \quad s \in S .
\end{aligned}
$$

Theorem: The target space $(\mathfrak{M}, \mathfrak{g})$ is locally symmetric if and only if $\left\langle L^{s}, L^{r}>_{\hat{G}}\left(L^{s}-\right.\right.$ $\left.L^{r}\right)=0$.

Proof: Let $\mathfrak{R} i e m$ denote the Riemann tensor of $(\mathfrak{M}, \mathfrak{g})$. The latter is locally symmetric, if and only if

$$
\nabla \Re \text { iem }=0,
$$

where $\nabla$ denotes the covariant derivative w.r.t $\mathfrak{g}$. However, the only non-trivial equations (6.3) are

$$
\nabla_{p} \Re_{s r q A}=k_{p s r q}<L^{s}, L^{r}>_{\hat{G}}\left(L_{A}^{r}-L_{A}^{s}\right)=0, \quad A=1, \ldots, N, \quad p, q, r, s \in S
$$

with $k_{p s r q}:=\varepsilon_{s} \varepsilon_{r} e^{2 U^{s}+2 U^{r}}\left(\delta_{p s} \delta_{r q}+\delta_{p r} \delta_{s q}\right)$ nonzero for fixed $s, r$.

\section{Scalar plus $p$-branes with spherical symmetry}

let us now examine static, spherically symmetric, multidimensional space-times with

$$
M=M_{-1} \times M_{0} \times M_{1} \times \cdots \times M_{N}, \quad \operatorname{dim} M_{i}=d_{i}, \quad i=0, \ldots, N,
$$

where $M_{-1} \subset \mathbb{R}$ corresponds to a radial coordinate $u, M_{0}=S^{2}$ is a 2-sphere, $M_{1} \subset \mathbb{R}$ is time, and $M_{i}, i>1$ are internal factor spaces. The metric is assumed correspondingly to be

$$
\begin{aligned}
d s^{2} & =\mathrm{e}^{2 \alpha(u)} d u^{2}+\sum_{i=0}^{N} \mathrm{e}^{2 \beta_{i}(u)} d s_{i}^{2} \\
& \equiv-\mathrm{e}^{2 \gamma(u)} d t^{2}+\mathrm{e}^{2 \alpha(u)} d u^{2}+\mathrm{e}^{2 \beta_{0}(u)} d \Omega^{2}+\sum_{i=2}^{N} \mathrm{e}^{2 \beta_{i}(u)} d s_{i}^{2}
\end{aligned}
$$


where $d s_{0}^{2} \equiv d \Omega^{2}=d \theta+\sin ^{2} \theta d \phi^{2}$ is the line element on $S^{2}, d s_{1}^{2} \equiv-d t^{2}$ with $\beta_{1}=: \gamma$, and $d s_{i}^{2}$, $i>1$, are $u$-independent line elements of internal Ricci-flat spaces of arbitrary dimensions $d_{i}$ and signatures $\varepsilon_{i}$.

For simplicity here let us only consider a single scalar field denoted as $\varphi$.

An electric-type $p+2$-form $F_{\mathrm{e} I}$ has a domain given by a product manifold

$$
M_{I}=M_{i_{1}} \times \cdots \times M_{i_{k}}
$$

where

$$
I=\left\{i_{1}, \ldots, i_{k}\right\} \subset I_{0} \stackrel{\text { def }}{=}\{0,1, \ldots, N\} .
$$

The corresponding dimensions are

$$
d(I) \stackrel{\text { def }}{=} \sum_{i \in I} d_{i}, \quad d\left(I_{0}\right)=D-1
$$

A magnetic-type $F$-form of arbitrary rank $k$ may be defined as a form on a domain $M_{\bar{I}}$ with $\bar{I} \stackrel{\text { def }}{=} I_{0}-I$, dual to an electric-type form,

$$
F_{\mathrm{m} I, M_{1} \ldots M_{k}}=\mathrm{e}^{-2 \lambda \varphi}(* F)_{\mathrm{e} I, M_{1} \ldots M_{k}} \equiv \mathrm{e}^{-2 \lambda \varphi} \frac{\sqrt{g}}{k !} \varepsilon_{M_{1} \ldots M_{k} N_{1} \ldots N_{D-k}} F_{\mathrm{e} I}^{N_{1} \ldots N_{D-k}},
$$

where $*$ is the Hodge operator and $\varepsilon$ is the totally antisymmetric Levi-Civita symbol.

For simplicity we now considering a just a single $n$-form, i.e. a single electric type and a single dual magnetic component, whence

$$
\operatorname{rank} F_{\mathrm{m} I}=D-\operatorname{rank} F_{\mathrm{e} I}=d(\bar{I}),
$$

whence $k=n$ in $(7.6)$ and

$$
d(I)=n-1 \quad \text { for } \quad F_{\mathrm{e} I}, \quad d(I)=d\left(I_{0}\right)-n=D-n-1 \quad \text { for } \quad F_{\mathrm{m} I} .
$$

All fields must be compatible with spherical symmetry and staticity. Correspondingly, the vector $\varphi$ of scalars and the $p+2$-forms valued fields depend (besides on their domain as forms) on the radial variable $u$ only.

Furthermore, the domain of the electric form $F_{\mathrm{e} I}$ does not include the sphere $M_{0}=S^{2}$, and $F_{\mathrm{e} I}$ is specified by a $u$-dependent potential form,

$$
F_{\mathrm{e} I, u L_{2} \ldots L_{n}}=\partial_{[u} U_{\left.L_{2} \ldots L_{n}\right]} \quad U=U_{L_{2}, \ldots, L_{n}} d x^{L_{2}} \wedge \ldots \wedge d x^{L_{n}} .
$$


Since the time manifold $M_{1}$ is a factor space of $M_{I}$, the form $(\sqrt{7.9})$ describes an electric $(n-2)$ brane in the remaining subspace of $M_{I}$. Similarly (7.6) describes a magnetic $(D-n-2)$-brane in $M_{I}$.

Let us label all nontrivial components of $F$ by a collective index $s=\left(I_{s}, \chi_{s}\right)$, where $I=$ $I_{s} \subset I_{0}$ characterizes the subspace of $M$ as described above and $\chi_{s}= \pm 1$ according to the rule

$$
\mathrm{e} \mapsto \chi_{s}=+1, \quad \mathrm{~m} \mapsto \chi_{s}=-1 .
$$

If $1 \in I$, the corresponding $p$-brane evolves with $t$ and we have a true electric or magnetic field, otherwise the potential (7.9) does not depend on $\bar{M}_{0}$, i.e. it is just a scalar in 4 dimensions. In this case we call the corresponding electric-type $F$ component (7.9) electric quasiscalar and its dual, magnetic-type, $F$ component (7.6) magnetic quasiscalar. So there are in general four types of $F$-field components (summarized in Table 四): electric (E), magnetic (M), electric quasiscalar (EQ), magnetic quasiscalar (MQ). The choice of subsets $I_{s}$ is only constrained

Table 1: Different types of antisymmetric $p+2$-form fields

\begin{tabular}{|l|lll|}
\hline E & electric $(1 \in I)$ & $F_{t u A_{3} \ldots A_{n}}$ & $A_{k}$ (coordinate) index of $M_{I}$ \\
M & magnetic $(1 \in I)$ & $F_{\theta \phi B_{3} \ldots B_{n}}$ & $B_{l}$ (coordinate) index of $M_{\bar{I}}$ \\
EQ & electric quasiscalar $(1 \notin I)$ & $F_{u A_{2} \ldots A_{n}}$ & $A_{k}$ (coordinate) index of $M_{I}$ \\
MQ & magnetic quasiscalar $(1 \notin I)$ & $F_{t \theta \phi B_{4} \ldots B_{n}}$ & $B_{l}$ (coordinate) index of $M_{\bar{I}}$ \\
\hline
\end{tabular}

by the multidimensional decomposition condition 3.11 for the energy-momentum tensor. Since antisymmetric $p+2$-form field components of type E and M (and type EQ and MQ respectively) just complement each other, they should be considered as independent of each other. In the following we consider all $F_{s}$ as independent fields (up to index permutations) each with a single nonzero component.

Let us assume Ricci-flat internal spaces. With spherical symmetry and staticity all field become independent of $M_{0}$ and $M_{0}$ respectively. And the variation reduces further from $\bar{M}_{0}$ to the radial manifold $M_{-1}$.

The reparametrization gauge on the lower dimensional manifold here is chosen as the (generalized) harmonic one [13]. Since $M_{-1}$ is 1-dimensional $u$ is a harmonic coordinate, $\square u=0$, such that

$$
\alpha(u)=\sigma_{0}(u)
$$


The nonzero Ricci tensor components are

$$
\begin{aligned}
\mathrm{e}^{2 \alpha} R_{t}^{t} & =-\gamma^{\prime \prime}, \\
\mathrm{e}^{2 \alpha} R_{u}^{u} & =-\alpha^{\prime \prime}+\alpha^{\prime 2}-\gamma^{\prime 2}-2{\beta^{\prime}}^{2}-\sum_{i=2}^{N} d_{i}{\beta_{i}^{\prime}}^{2}, \\
\mathrm{e}^{2 \alpha} R_{\theta}^{\theta} & =\mathrm{e}^{2 \alpha} R_{\phi}^{\phi}=\mathrm{e}^{2 \alpha-2 \beta}-\beta^{\prime \prime}, \\
\mathrm{e}^{2 \alpha} R_{a_{j}}^{b_{i}} & =-\delta_{a_{j}}^{b_{i}} \beta_{i}^{\prime \prime} \quad(i, j=1, \ldots, N),
\end{aligned}
$$

where a prime denotes $d / d u$ and the indices $a_{i}, b_{i}$ belong to the $i$-th internal factor space. The Einstein tensor component $G_{1}^{1}$ does not contain second-order derivatives:

$$
\mathrm{e}^{2 \alpha} G_{1}^{1}=-\mathrm{e}^{2 \alpha-2 \beta}+\frac{1}{2} \alpha^{\prime 2}-\frac{1}{2}\left({\gamma^{\prime}}^{2}+2{\beta^{\prime}}^{2}+\sum_{i=2}^{N} d_{i} \beta_{i}^{\prime 2}\right) .
$$

The corresponding component of the Einstein equations is an integral of other components, similar to the energy integral in cosmology.

The generalized Maxwell equations give

$$
\begin{aligned}
F_{\mathrm{e} I}^{u M_{2} \ldots M_{n}} & =Q_{\mathrm{e} I} \mathrm{e}^{-2 \alpha-2 \lambda \varphi}, & & Q_{\mathrm{e} I}=\text { const } \\
F_{\mathrm{m} I, u M_{1} \ldots M_{d(\bar{I})}} & =Q_{\mathrm{m} I} \sqrt{\left|g_{\bar{I}}\right|}, & & Q_{\mathrm{m} I}=\mathrm{const}
\end{aligned}
$$

where $\left|g_{\bar{I}}\right|$ is the determinant of the $u$-independent part of the metric of $M_{\bar{I}}$ and $Q_{s}$ are charges. These solutions provide then the energy momentum tensors, of the electric and magnetic $p+2-$ forms written in matrix form,

$$
\begin{aligned}
& \mathrm{e}^{2 \alpha}\left(T_{M}^{N}\left[F_{\mathrm{e} I}\right]\right)=-\frac{1}{2} \eta_{F} \varepsilon(I) Q_{\mathrm{e} I}^{2} \mathrm{e}^{2 y_{\mathrm{e} I}} \operatorname{diag}\left(+1,[1]_{I},[-1]_{\bar{I}}\right) \\
& \mathrm{e}^{2 \alpha}\left(T_{M}^{N}\left[F_{\mathrm{m} I}\right]\right)=\frac{1}{2} \eta_{F} \varepsilon(\bar{I}) Q_{\mathrm{m} I}^{2} \mathrm{e}^{2 y_{\mathrm{m} I}} \operatorname{diag}\left(1,[1]_{I},[-1]_{\bar{I}}\right),
\end{aligned}
$$

where the first position belongs to $u$ and $f$ operating over $M_{J}$ is denoted by $[f]_{J}$. The functions $y_{s}(u)$ are

$$
y_{s}(u)=\sigma\left(I_{s}\right)-\chi_{s} \lambda \varphi
$$

The scalar field EMT is

$$
\mathrm{e}^{2 \alpha} T_{M}^{N}[\varphi]=\frac{1}{2}\left(\varphi^{a}\right)^{\prime 2} \operatorname{diag}\left(+1,[-1]_{I_{0}}\right)
$$


The sets $I_{s} \in I_{0}$ may be classified by types E, M, EQ, MQ according to the description in the previous section. Denoting $I_{s}$ for the respective types by $I_{\mathrm{E}}, I_{\mathrm{M}}, I_{\mathrm{EQ}}, I_{\mathrm{MQ}}$, we see from (7.16) that, positive electric and magnetic energy densities require

$$
\eta_{f}=-\varepsilon\left(I_{\mathrm{E}}\right)=\varepsilon\left(\bar{I}_{\mathrm{M}}\right)=\varepsilon\left(I_{\mathrm{EQ}}\right)=-\varepsilon\left(\bar{I}_{\mathrm{MQ}}\right) .
$$

If $t$ is the only time coordinate, (7.19) with $\eta_{F}=1$ holds for any choices of $I_{s}$. If there exist other times, then the relations (7.19) constrain the subspaces where the different $F$ components may be specified.

Since the total EMT on the r.h.s. of the Einstein equations has the property

$$
T_{u}^{u}+T_{\theta}^{\theta}=0,
$$

the corresponding combination on the l.h.s becomes an integrable Liouville form

$$
\begin{aligned}
G_{u}^{u}+G_{\theta}^{\theta} & =\mathrm{e}^{-2 \alpha}\left[-\alpha^{\prime \prime}+\beta_{0}^{\prime \prime}+\mathrm{e}^{2 \alpha-2 \beta_{0}}\right]=0, \\
\mathrm{e}^{\beta_{0}-\alpha} & =s(k, u),
\end{aligned}
$$

where $k$ is an integration constant (IC) and the function $s(k,$.$) is defined as follows:$

$$
s(k, u) \stackrel{\text { def }}{=} \begin{cases}k^{-1} \sinh k u, & k>0 \\ u, & k=0 \\ k^{-1} \sin k u, & k<0\end{cases}
$$

Another IC is suppressed by adjusting the origin of the $u$ coordinate.

With (7.21) the $D$-dimensional line element may be written in the form

$$
d s^{2}=\frac{\mathrm{e}^{-2 \sigma_{1}}}{s^{2}(k, u)}\left[\frac{d u^{2}}{s^{2}(k, u)}+d \Omega^{2}\right]+\sum_{i=1}^{N} \mathrm{e}^{2 \beta_{i}} d s_{i}^{2}
$$

where $\sigma_{1}$ has been defined in (2.10).

Let us treat the whole set of unknowns $\beta_{i}(u), \varphi(u)$ as a real-valued vector function $x(u)$ in an $(N+1)$-dimensional vector space $V$, with components $x^{A}=\beta_{A}$ for $A=1, \ldots, N$ and $x^{N+1}=\varphi$.

Then the field equations for $\beta_{i}$ and $\varphi$ coincide with the equations of motion corresponding to the Lagrangian of a Euclidean Toda-like system

$$
L=\bar{G}_{A B} x^{\prime A} x^{\prime B}-V_{Q}(y), \quad V_{Q}(y)=\sum_{s} \theta_{s} Q_{s}^{2} \mathrm{e}^{2 y_{s}},
$$


where, according to (7.19), $\theta_{s}=1$ if $F_{s}$ is a true electric or magnetic field and $\theta_{s}=-1$ if $F_{s}$ is quasiscalar. The nondegenerate, symmetric matrix

$$
\left(\bar{G}_{A B}\right)=\left(\begin{array}{cc}
G_{i j} & 0 \\
0 & 1
\end{array}\right), \quad G_{i j}=d_{i} d_{j}+d_{i} \delta_{i j}
$$

defines a positive-definite metric in $V$. The energy constraint corresponding to (7.24) is

$$
E=\sigma_{1}^{\prime 2}+\sum_{i=1}^{N} d_{i}{\beta^{\prime}}_{i}^{2}+\varphi^{\prime 2}+V_{Q}(y)=\bar{G}_{A B} x^{\prime A} x^{\prime B}+V_{Q}(y)=2 k^{2} \operatorname{sign} k
$$

with $k$ from (7.21). The integral (7.26) follows here from the $(u u)$ - component of (3.4).

The functions $y_{s}(u)(7.17)$ can be represented as scalar products in $V$ (recall that $s=$ $\left.\left(I_{s}, \chi_{s}\right)\right)$ :

$$
y_{s}(u)=Y_{s, A} x^{A}, \quad\left(Y_{s, A}\right)=\left(d_{i} \delta_{i I_{s}}, \quad-\chi_{s} \lambda\right)
$$

where $\delta_{i I}:=\sum_{j \in I} \delta_{i j}$ is an indicator for $i$ belonging to $I$ (1 if $i \in I$ and 0 otherwise).

The contravariant components of $Y_{s}$ are found using the matrix $\bar{G}^{A B}$ inverse to $\bar{G}_{A B}$ :

$$
\begin{aligned}
& \left(\bar{G}^{A B}\right)=\left(\begin{array}{cc}
G^{i j} & 0 \\
0 & 1
\end{array}\right), \quad G^{i j}=\frac{\delta^{i j}}{d_{i}}-\frac{1}{D-2} \\
& \left(Y_{s}^{A}\right)=\left(\delta_{i I_{s}}-\frac{d\left(I_{s}\right)}{D-2},-\chi_{s} \lambda\right),
\end{aligned}
$$

and the scalar products of different $Y_{s}$, whose values are of primary importance for the integrability of our system, are

$$
Y_{s, A} Y_{s^{\prime}}{ }^{A}=d\left(I_{s} \cap I_{s^{\prime}}\right)-\frac{d\left(I_{s}\right) d\left(I_{s^{\prime}}\right)}{D-2}+\chi_{s} \chi_{s^{\prime}} \lambda^{2}
$$

\section{Purely EM black hole solutions}

In [19 it was shown that quasiscalar components of the $F$-fields are incompatible with orthobrane black holes. Therefore let us now consider only two $F$-field components, Type E and Type $\mathrm{M}$ according to the classification above. They will be electric as $F_{\mathrm{e}}$ and $F_{\mathrm{m}}$ and the corresponding sets $I_{s} \subset I_{0}$ as $I_{\mathrm{e}}$ and $I_{\mathrm{m}}$. Then a minimal configuration (7.1) of the manifold $M$ compatible with an arbitrary choice of $I_{s}$ has the following form: 


$$
N=5, \quad I_{0}=\{0,1,2,3,4,5\}, \quad I_{e}=\{1,2,3\}, \quad I_{m}=\{1,2,4\},
$$

so that

$$
\begin{array}{ll}
\left(I_{0}\right)=D-1, & d\left(I_{\mathrm{e}}\right)=n-1, \quad d\left(I_{\mathrm{m}}\right)=D-n-1, \quad d\left(I_{\mathrm{e}} \cap I_{\mathrm{m}}\right)=1+d_{2} ; \\
d_{1}=1, & d_{2}+d_{3}=d_{3}+d_{5}=n-2 .
\end{array}
$$

The relations (8.2) show that, given $D$ and $d_{2}$, all $d_{i}$ are known.

This corresponds to an electric $(n-2)$-brane located on the subspace $M_{2} \times M_{3}$ and a magnetic $(D-n-2)$-brane on the subspace $M_{2} \times M_{4}$. Their intersection dimension $d_{\text {int }}=d_{2}$ turns out to determine qualitative properties of the solutions.

The index $s$ now takes the two values e and $\mathrm{m}$ and

$$
\begin{aligned}
Y_{\mathrm{e}, A} & =\left(1, d_{2}, d_{3}, 0,0,-\lambda\right) ; \\
Y_{\mathrm{m}, A}=\left(1, d_{2}, 0, d_{4}, 0, \lambda\right) ; & \\
Y_{\mathrm{e}}^{A} & =(1,1,1,0,0,-\lambda)-\frac{n-1}{D-2}(1,1,1,1,1,0) ; \\
Y_{\mathrm{m}}^{A} & =(1,1,0,1,0, \lambda)-\frac{D-n-1}{D-2}(1,1,1,1,1,0),
\end{aligned}
$$

where the last component of each vector refers to $x^{N+1}=x^{6}=\varphi$.

In the solutions presented below the set of ICs will be reduced by the condition that the space-time be asymptotically flat at spatial infinity $(u=0)$ and by a choice of scales in the relevant directions. Namely, we put

$$
\beta_{i}(0)=0=\varphi(0) \quad i=1,2,3,4,5 .
$$

The requirement $\varphi(0)=0$ is convenient and may be always satisfied by a redefinition of the charges. The conditions $\beta_{i}(0)=0(i>1)$ mean that the real scales of the extra dimensions are hidden in the internal metrics $d s_{i}^{2}$ independent of whether or not they are assumed to be compact.

In the following, both cases, orthobrane solutions and solutions with degenerate charges, are considered first generally and then for the minimal configuration (8.1)-(8.4).

\section{VIII.1 Orthobrane black hole solutions}

Assuming that the vectors $Y_{s}$ are mutually orthogonal with respect to the metric $\bar{G}_{A B}$, i.e.

$$
Y_{s, A} Y_{s^{\prime}}{ }^{A}=\delta_{s s^{\prime}} N_{s}^{2}
$$


the number of functions $y_{s}$ does not exceed the number of equations, and the system becomes integrable. Due to (7.8), the norms $N_{s}$ are actually $s$-independent:

$$
N_{s}^{2}=d\left(I_{s}\right)\left[1-\frac{d\left(I_{s}\right)}{D-2}\right]+\lambda^{2}=\frac{(n-1)(D-n-1)}{D-2}+\lambda^{2} \stackrel{\text { def }}{=} \frac{1}{\nu},
$$

$\nu>0$.

Due to 8.5$)$, the functions $y_{s}(u)$ obey the decoupled equations

$$
y_{s}^{\prime \prime}=\theta_{s} \frac{Q_{s}^{2}}{\nu} \mathrm{e}^{2 y_{s}}
$$

whence

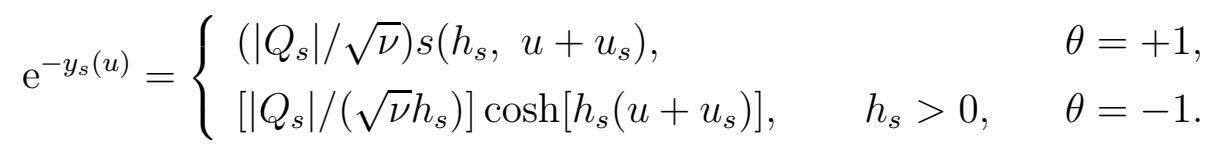

where $h_{s}$ and $u_{s}$ are ICs and the function $s$ was defined in $(\overline{7.22})$. For the functions $x^{A}(u)$ we obtain:

$$
x^{A}(u)=\nu \sum_{s} Y_{s}^{A} y_{s}(u)+c^{A} u+\bar{c}^{A},
$$

where the vectors of ICs $c^{A}$ and $\bar{c}^{A}$ satisfy the orthogonality relations $c^{A} Y_{s, A}=\bar{c}^{A} Y_{s, A}=0$, or

$$
c^{i} d_{i} \delta_{i I_{s}}-\lambda c^{N+1} \chi_{s}=0, \quad \quad \bar{c}^{i} d_{i} \delta_{i I_{s}}-\lambda \bar{c}^{N+1} \chi_{s}=0 .
$$

Specifically, the logarithms of the scale factors $\beta_{i}$ and the scalar field $\varphi$ are

$$
\begin{aligned}
\beta_{i}(u) & =\nu \sum_{s}\left[\delta_{i I_{s}}-\frac{d\left(I_{s}\right)}{D-2}\right] y_{s}(u)+c^{i} u+\bar{c}^{i}, \\
\varphi(u) & =-\lambda \nu \sum_{s} y_{s}(u)+c^{N+1} u+\bar{c}^{N+1},
\end{aligned}
$$

and the function $\sigma_{1}$ which appears in the metric (7.23) is

$$
\sigma_{1}=-\frac{\nu}{D-2} \sum_{s} d\left(I_{s}\right) y_{s}(u)+c^{0} u+\bar{c}^{0}
$$

with

$$
c^{0}=\sum_{i=1}^{N} d_{i} c^{i}, \quad \bar{c}^{0}=\sum_{i=1}^{N} d_{i} \bar{c}^{i}
$$


Finally, (7.26) now reads

$$
E=\nu \sum_{s} h_{s}^{2} \operatorname{sign} h_{s}+\bar{G}_{A B} c^{A} c^{B}=2 k^{2} \operatorname{sign} k .
$$

The relations (7.11), (7.14), (7.15), (7.21), (7.23), 8.8)-(8.15), along with the definitions (7.22) and (8.6) and the restriction (8.5), entirely determine the general solution.

For the minimal configuration (8.1)-(8.4), the orthogonality condition (8.5) reads

$$
\lambda^{2}=d_{2}+1-\frac{1}{D-2}(n-1)(D-n-1)
$$

In particular, in dilaton gravity $n=2, d_{2}=0$ and the integrability condition (8.16) just reads $\lambda^{2}=1 /(D-2)$, which is a well-known relation of string gravity. The familiar ReissnerNordström solution, $D=4, n=2, \lambda=0, d_{2}=0$ does not satisfy Eq. (8.16). (It will be recovered indeed as a degenerate case below.) Some examples of configurations satisfying the orthogonality condition (8.16) in the purely topological case $\lambda=0$ are summarized in Table 2 (including the values of the constants $B$ and $C$ from (8.28) ). In this case (8.16) is just a Diophantus equation for $D, n$ and $d_{2}$.

Table 2: Orthobrane solutions with $\lambda=0$

\begin{tabular}{|c|l|l|l|l|l|l|}
\hline & $n$ & $d\left(I_{\mathrm{e}}\right)$ & $d\left(I_{\mathrm{m}}\right)$ & $d_{2}$ & $B$ & $C$ \\
\hline $\begin{array}{l}D=4 m+2 \\
(m \in \mathbb{N})\end{array}$ & $2 \mathrm{~m}+1$ & $2 \mathrm{~m}$ & $2 \mathrm{~m}$ & $\mathrm{~m}-1$ & $1 / \mathrm{m}$ & $1 / \mathrm{m}$ \\
$\mathrm{D}=11$ & 4 & 3 & 6 & 1 & $2 / 3$ & $1 / 3$ \\
& 7 & 6 & 3 & 1 & $1 / 3$ & $2 / 3$ \\
\hline
\end{tabular}

The solution is entirely determined by inserting (8.3) into (8.9) with $\bar{c}^{A}=0$ due to (8.4),

$$
x^{A}(u)=\nu \sum_{s} Y_{s}^{A} y_{s}(u)+c^{A} u ; \quad \mathrm{e}^{-y_{s}(u)}=\left(\left|Q_{s}\right| / \sqrt{\nu}\right) s\left(h_{s}, u+u_{s}\right) .
$$


Due to 8.16 ) the parameter $\nu$ is

$$
\nu=1 / \sqrt{1+d_{2}} \text {. }
$$

The constants are connected by the relations

$$
\begin{aligned}
& \left(\left|Q_{\mathrm{e}, \mathrm{m}}\right| / \nu\right) s\left(h_{\mathrm{e}, \mathrm{m}}, u_{\mathrm{e}, \mathrm{m}}\right)=1 ; \\
& c^{1}+d_{2} c^{2}+d_{3} c^{3}-\lambda c^{6}=0 ; \\
& \frac{h_{\mathrm{e}}^{2} \operatorname{sign} h_{\mathrm{e}}+h_{\mathrm{m}}^{2} \operatorname{sign} h_{\mathrm{m}}}{1+d_{2}}+G_{i j} c^{i} c^{j}+\left(c^{6}\right)^{2}=2 k^{2} \operatorname{sign} k,
\end{aligned}
$$

where the matrix $G_{i j}$ is given in (7.25) and all $\bar{c}^{A}=0$ due to the boundary conditions (8.4). The fields $\varphi$ and $F$ are given by Eqs. (7.14), (7.15), (8.12).

This solution contains 8 nontrivial, independent ICs, namely, $Q_{\mathrm{e}}, Q_{\mathrm{m}}, h_{\mathrm{e}}, h_{\mathrm{m}}$ and 4 others from the set $c^{A}$ constrained by (8.19).

For black holes, we require that all $\left|\beta_{i}\right|<\infty, i=2, \ldots, N$ (regularity of extra dimensions), $|\varphi|<\infty$ (regularity of the scalar field) and $\left|\beta_{0}\right|<\infty$ (finiteness of the spherical radius) as $u \rightarrow \infty$. With $y_{s}(u) \sim-h_{s} u$, this leads to the following constraints on the ICs:

$$
c^{A}=-k \sum_{s}\left(\delta_{1 I_{s}}+\nu Y_{s}^{A} h_{s}\right)
$$

where $A=1$ corresponds to $i=1$. Via orthonormality relations (8.10) for $c^{A}$, we obtain

$$
\begin{aligned}
& h_{s}=k \delta_{1 I_{s}}, \\
& c^{A}=-k \delta_{1}^{A}+k \nu \sum_{s} \delta_{1 I_{s}} Y_{s}^{A},
\end{aligned}
$$

and 8.15$)$ then holds automatically.

Let us now consider the case where (8.21) and (8.22) with $\delta_{1 I_{s}}=1$ hold. After a transformation $u \mapsto R$, to isotropic coordinates given by the relation

$$
\mathrm{e}^{-2 k u}=1-2 k / R,
$$

we obtain

$$
\begin{aligned}
& d s^{2}=-\frac{1-2 k / R}{P_{\mathrm{e}}^{B} P_{\mathrm{m}}^{C}} d t^{2}+P_{\mathrm{e}}^{C} P_{m}^{B}\left(\frac{d R^{2}}{1-2 k / R}+R^{2} d \Omega^{2}\right)+\sum_{i=2}^{5} \mathrm{e}^{2 \beta_{i}(u)} d s_{i}^{2}, \\
& \mathrm{e}^{2 \beta_{2}}=P_{\mathrm{e}}^{-B} P_{\mathrm{m}}^{-C}, \quad \mathrm{e}^{2 \beta_{3}}=\left(P_{\mathrm{m}} / P_{\mathrm{e}}\right)^{B},
\end{aligned}
$$




$$
\begin{aligned}
& \mathrm{e}^{2 \beta_{4}}=\left(P_{\mathrm{e}} / P_{\mathrm{m}}\right)^{C}, \quad \mathrm{e}^{2 \beta_{5}}=P_{\mathrm{e}}^{C} P_{\mathrm{m}}^{B}, \\
& e^{2 \lambda \varphi}=\left(P_{\mathrm{e}} / P_{\mathrm{m}}\right)^{2 \lambda^{2} /\left(1+d_{2}\right)}, \\
& F_{01 M_{3} \ldots M_{n}}=-Q_{\mathrm{e}} /\left(R^{2} P_{\mathrm{e}}\right), \quad F_{23 M_{3} \ldots M_{n}}=Q_{\mathrm{m}} \sin \theta,
\end{aligned}
$$

with the notations

$$
\begin{aligned}
& P_{\mathrm{e}, \mathrm{m}}=1+p_{\mathrm{e}, \mathrm{m}} / R, \quad p_{\mathrm{e}, \mathrm{m}}=\sqrt{k^{2}+\left(1+d_{2}\right) Q_{\mathrm{e}, \mathrm{m}}^{2}}-k ; \\
& B=\frac{2(D-n-1)}{(D-2)\left(1+d_{2}\right)}, \quad C=\frac{2(n-1)}{(D-2)\left(1+d_{2}\right)} .
\end{aligned}
$$

The BH gravitational mass as determined from a comparison of (8.24) with the Schwarzschild metric for $R \rightarrow \infty$ is

$$
G_{N} M=k+\frac{1}{2}\left(B p_{\mathrm{e}}+C p_{\mathrm{m}}\right)
$$

where $G_{N}$ is the Newtonian gravitational constant. This expression, due to $k>0$, provides a restriction upon the charge combination for a given mass, namely,

$$
B\left|Q_{\mathrm{e}}\right|+C\left|Q_{\mathrm{m}}\right|<2 G_{N} M / \sqrt{1+d_{2}}
$$

The inequality is replaced by equality in the extreme limit $k=0$. For $k=0$ our BH turns into a naked singularity (at the centre $R=0$ ) for any $d_{2}>0$, while for $d_{2}=0$ the zero value of $R$ is not a centre $\left(g_{22} \neq 0\right)$ but a horizon. In the latter case, if $\left|Q_{e}\right|$ and $\left|Q_{m}\right|$ are different, the remaining extra-dimensional scale factors are smooth functions for all $R \geq 0$.

For a static, spherical BH one can define a Hawking temperature $T_{H}:=\kappa / 2 \pi$ as given by the surface gravity $\kappa$. With a generalized Komar integral (see e.g. [20])

$$
M(r):=-\frac{1}{8 \pi} \int_{S_{r}} * d \xi
$$

over the time-like Killing form $\xi$, the surface gravity can be evaluated as

$$
\kappa=M\left(r_{H}\right) /\left(r_{H}\right)^{2}=\left(\sqrt{\left|g_{00}\right|}\right)^{\prime} /\left.\sqrt{g_{11}}\right|_{r=r_{H}}=\left.\mathrm{e}^{\gamma-\alpha}\left|\gamma^{\prime}\right|\right|_{r=r_{H}},
$$

where a prime, $\alpha$, and $\gamma$ are understood in the sense of the general metric (7.2) and $k_{\mathrm{B}}$ is the Boltzmann constant. The expression (8.32) is invariant with respect to radial coordinate reparametrization, as is necessary for any quantity having a direct physical meaning. It is also invariant under conformal mappings with a conformal factor which is smooth at the horizon. 
Substituting $g_{00}$ and $g_{11}$ from (8.24), one obtains:

$$
T_{H}=\frac{1}{2 \pi k_{\mathrm{B}}} \frac{1}{4 k}\left[\frac{4 k^{2}}{\left(2 k+p_{\mathrm{e}}\right)\left(2 k+p_{\mathrm{m}}\right)}\right]^{1 /\left(d_{2}+1\right)} .
$$

If $d_{2}=0$ and both charges are nonzero, this temperature tends to zero in the extreme limit $k \rightarrow 0$; if $d_{2}=1$ and both charges are nonzero, it tends to a finite limit, and in all other cases it tends to infinity. Remarkably, it is determined by the $p$-brane intersection dimension $d_{2}$ rather than the whole space-time dimension $D$.

\section{VIII.2 The solution for $Q_{\mathrm{e}}^{2}=Q_{\mathrm{m}}^{2}$}

In this degenerate case, solutions can be found which need not satisfy the orthobrane condition (8.5). Let us suppose that two functions (7.17), say, $y_{1}$ and $y_{2}$, coincide up to an addition of a constant (which may be then absorbed by re-defining a charge $Q_{1}$ or $Q_{2}$ ) while corresponding vectors $Y_{1}$ and $Y_{2}$ are neither coinciding, nor orthogonal (otherwise we would have the previously considered situation). Substituting $y_{1} \equiv y_{2}$ into (7.27), one obtains

$$
\left(Y_{1, A}-Y_{2, A}\right) x^{A}=0 .
$$

This is a constraint reducing the number of independent unknowns $x^{A}$. Furthermore, substituting (8.34) to the Lagrange equations for $x^{A}$,

$$
-\left(Y_{1, A}-Y_{2, A}\right) x^{\prime \prime A}=\sum_{s} \theta_{s} Q_{s}^{2} \mathrm{e}^{2 y_{s}} Y_{s}^{A}\left(Y_{1, A}-Y_{2, A}\right)=0 .
$$

In this sum all coefficients of different functions $\mathrm{e}^{2 y_{s}}$ must be zero. This yields new orthogonality conditions

$$
Y_{s}^{A}\left(Y_{1, A}-Y_{2, A}\right)=0, \quad s \neq 1,2,
$$

now for the difference $Y_{1}-Y_{2}$ and other $Y_{s}$, and with Eq. (8.6) the relation

$$
\left(\nu^{-1}-Y_{1}^{A} Y_{2, A}\right)\left(\theta_{1} Q_{1}^{2}-\theta_{2} Q_{2}^{2}\right)=0
$$

The first multiplier in (8.37) is positive $\left(\bar{G}_{A B}\right.$ is positive-definite, hence a scalar product of two different vectors with equal norms is smaller than their norm squared). Therefore

$$
\theta_{1}=\theta_{2}, \quad Q_{1}^{2}=Q_{2}^{2}
$$

Imposing the constraints (8.34), (8.36), (8.38), reduces the numbers of unknowns and integration constants, and simultaneously also reduces the number of restrictions on the input 
parameters (by the orthogonality conditions (8.5)). Due to (8.38), this is only possible when the two components with coinciding charges are of equal nature: both must be either true electric/magnetic ones $\left(\theta_{s}=1\right)$, or quasiscalar ones $\left(\theta_{s}=-1\right)$. Correspondingly, we now set $y(u):=y_{\mathrm{e}}=y_{\mathrm{m}}$ and $Q^{2}:=Q_{\mathrm{e}}^{2}=Q_{\mathrm{m}}^{2}$.

For the minimal configuration (8.1)-(8.3), eq. (8.34) yields

$$
d_{3} \beta_{3}-d_{4} \beta_{4}-2 \lambda \varphi=0 .
$$

Eqs. (8.36) are irrelevant here since we are dealing with two functions $y_{s}$ only. The equations of motion for $x^{A}$ now take the form

$$
x^{A^{\prime \prime}}=Q^{2} \mathrm{e}^{2 y}\left(Y_{\mathrm{e}}^{A}+Y_{\mathrm{m}}^{A}\right) .
$$

Their proper combination gives $y^{\prime \prime}=\left(1+d_{2}\right) Q^{2} \mathrm{e}^{2 y}$, whence

$$
\mathrm{e}^{-y}=\sqrt{\left(1+d_{2}\right) Q^{2}} s\left(h, u+u_{1}\right)
$$

where the function $s$ is defined in (7.22) and $h, u_{1}$ are ICs and, due to (8.4), $\sqrt{\left(1+d_{2}\right) Q^{2}} s\left(h, u_{1}\right)=1$. Other unknowns are easily determined using (8.40) and (8.4):

$$
\begin{aligned}
x^{A} & =\nu Y^{A} y+c^{A} ; \quad Y^{A}=Y_{\mathrm{e}}^{A}+Y_{\mathrm{m}}^{A}=(1,1,0,0,-1,0) ; \\
\sigma_{1} & =-\nu y+c_{0} u .
\end{aligned}
$$

Here, as in (8.18), $\nu=1 /\left(1+d_{2}\right)$, but it is now just a notation. The constants $c_{0}, h, c^{A}(A=$ $1, \ldots, 6)$ and $k$ (see (7.21)) are related by

$$
\begin{aligned}
& -c^{0}+\sum_{i=1}^{5} d_{i} c^{i}=0, \quad c^{1}+d_{2} c^{2}+d_{3} c^{3}-\lambda c^{6}=0, \quad c^{1}+d_{2} c^{2}+d_{4} c^{4}+\lambda c^{6}=0, \\
& 2 k^{2} \operatorname{sign} k=\frac{2 h^{2} \operatorname{sign} h}{1+d_{2}}\left(c^{0}\right)^{2}+\sum_{i=1}^{5} d_{i}\left(c^{i}\right)^{2}+\left(c^{6}\right)^{2} .
\end{aligned}
$$

Extra-dimensional scale factors remain finite as $u \rightarrow u_{\max }$ in the case of a BH. It is specified by the following values of the ICs:

$$
k=h>0, \quad c^{3}=c^{4}=c^{6}=0, \quad c_{2}=-c_{5}=-\frac{k}{1+d_{2}}, \quad c_{0}=c^{1}=-\frac{d_{2} k}{1+d_{2}} .
$$


The event horizon occurs at $u=\infty$. After the same transformation (8.23) the metric takes the form

$$
\begin{aligned}
d s_{D}^{2}=-\frac{1-2 k / R}{(1+p / R)^{2 \nu}} d t^{2} & +(1+p / R)^{2 \nu}\left(\frac{d R^{2}}{1-2 k / R}+R^{2} d \Omega^{2}\right) \\
& +(1+p / R)^{-2 \nu} d s_{2}^{2}+d s_{3}^{2}+d s_{4}^{2}+(1+p / R)^{2 \nu} d s_{5}^{2}
\end{aligned}
$$

with the notation

$$
p=\sqrt{k^{2}+\left(1+d_{2}\right) Q^{2}}-k
$$

The fields $\varphi$ and $F$ are determined by the relations

$$
\begin{gathered}
\varphi \equiv 0, \quad F_{01 L_{3} \ldots L_{n}}=-\frac{Q}{R^{2}(1+p / R)}, \quad F_{23 L_{3} \ldots L_{n}}=Q \sin \theta \\
G_{N} M=k+p /\left(1+d_{2}\right),
\end{gathered}
$$

The Hawking temperature can be calculated as before,

$$
T=\frac{1}{2 \pi k_{\mathrm{B}}} \frac{1}{4 k}\left(\frac{2 k}{2 k+p}\right)^{2 /\left(d_{2}+1\right)}
$$

The well-known results for the Reissner-Nordström metric are recovered when $d_{2}=0$. In this case $T \rightarrow 0$ in the extreme limit $k \rightarrow 0$. For $d_{2}=1, T$ tends to a finite limit as $k \rightarrow 0$ and for $d_{2}>1$ it tends to infinity. As is the case with two different charges, $T$ does not depend on the space-time dimension $D$, but depends on the $p$-brane intersection dimension $d_{2}$.

\section{The Einstein frame for dynamics and cosmology}

In this final section we discuss the issue of the physical frame for the particularly important case $D_{0}=4$. First of all, in this case a selfdual canonical formulation of dynamics is at hand, due to the particular spinor decomposition $\mathrm{so}(1,3)=\mathrm{su}(2) \oplus \mathrm{su}(2)$ of the tangent Lorentz symmetry. In the Einstein frame, the effective $\sigma$-model with $D_{0}=4$ admits in principle a canonical quantization of the geometry on $\bar{M}_{0}=\mathbb{R} \times M_{0}$ to the same extend and under the same assumptions as pure Einstein gravity does.

Since for a multidimensional geometry as defined above the imprint of the internal factor spaces is only by their scale factors, configuration space and phase space of such geometries will

only be extended by finite a finite number of dilatonic midisuperspace fields. However, only in 
the Einstein frame the coupling of the dilatonic fields to the $\bar{D}_{0}$-geometry will be minimal such that the quantization of the latter can be executed practically independently.

Let us denote the external space-time metric $\bar{g}^{(0)}$ in the Brans-Dicke frame with $\bar{\gamma} \stackrel{!}{=} 0$ as $\bar{g}^{(\mathrm{BD})}$ and in the Einstein frame with $f \stackrel{!}{=} 0$ as $\bar{g}^{(\mathrm{E})}$. It can be easily seen that they are connected with each other by a conformal transformation

$$
\bar{g}^{(\mathrm{E})} \mapsto \bar{g}^{(\mathrm{BD})}=\Omega^{2} \bar{g}^{(\mathrm{E})}
$$

with $\Omega$ from (2.39).

In particular, also for spatially homogeneous cosmological models (and with $t \leftrightarrow u$ for spherically symmetric static models) solutions have to be transformed to the Einstein frame before a physical interpretation can be given.

Under any projection $\mathrm{pr}_{0}: \overline{M_{0}} \rightarrow \mathbb{R}$ a consistent pullback of the metric $-e^{2 \gamma(\tau)} d \tau \otimes d \tau$ from $\tau \in \mathbb{R}$ to $x \in \operatorname{pr}_{0}^{-1}\{\tau\} \subset \bar{M}_{0}$ is given by

$$
\bar{g}^{(\mathrm{BD})}(x):=-e^{2 \gamma(\tau)} d \tau \otimes d \tau+e^{2 \beta^{0}(x)} g^{(0)} .
$$

In particular, for spatially (metrically-)homogeneous cosmological models all scale factors $a_{i}:=$ $e^{\beta^{i}}, i=0, \ldots, n$, depend only on $\tau \in \mathbb{R}$.

With (9.2) and (9.1), Eq. (2.6) reads

$$
\begin{aligned}
g & =-e^{2 \gamma(\tau)} d \tau \otimes d \tau+a_{0}^{2} g^{(0)}+\sum_{i=1}^{n} e^{2 \beta^{i}} g^{(i)} \\
& =-d t_{\mathrm{BD}} \otimes d t_{\mathrm{BD}}+a_{\mathrm{BD}}^{2} g^{(0)}+\sum_{i=1}^{n} e^{2 \beta^{i}} g^{(i)} \\
& =-\Omega^{2} d t_{\mathrm{E}} \otimes d t_{\mathrm{E}}+\Omega^{2} a_{\mathrm{E}}^{2} g^{(0)}+\sum_{i=1}^{n} e^{2 \beta^{i}} g^{(i)},
\end{aligned}
$$

where $a_{0}:=a_{\mathrm{BD}}$ and $a_{\mathrm{E}}$ are the external space scale factor functions depending respectively on the cosmic synchronous time $t_{\mathrm{BD}}$ and $t_{\mathrm{E}}$ in the Brans-Dicke and Einstein frame. With (2.39) the latter is related to the former by

$$
a_{\mathrm{E}}=\Omega^{-1} a_{\mathrm{BD}}=\left(\prod_{i=1}^{n} e^{d_{i} \beta^{i}}\right)^{\frac{1}{D_{0}-2}} a_{\mathrm{BD}},
$$

and the cosmic time of the Einstein frame is given by

$$
\pm d t_{\mathrm{E}}=\Omega^{-1} e^{\gamma} d \tau=\left(\prod_{i=1}^{n} e^{d_{i} \beta^{i}}\right)^{\frac{1}{D_{0}-2}} d t_{\mathrm{BD}} .
$$


Since $a_{\mathrm{BD}}^{2}\left(d \eta_{\mathrm{BD}}\right)^{2}=\Omega^{2} a_{\mathrm{E}}^{2}\left(d \eta_{\mathrm{E}}\right)^{2}$, the conformal times of the Einstein and the Brans-Dicke frame agree (up to time reversal). This has sometimes guided authors to compare the frames in conformal time (see e.g. [22]). However (at least for cosmology) the physical relevant time is the cosmic synchronous time, which is different for different frames, in particular for the Einstein and Brans-Dicke frame.

In [23] several reasons have been listed why minimal coupling between geometry and matter and hence the Einstein frame is the preferred choice. There also a general prescription for the transformation of well known solutions from the Brans-Dicke frame to the Einstein frame has been given. It was demonstrated explicitly that qualitative cosmological features change significantly under this transformation. This was shown for a couple of examples, including the general multidimensional Kasner solution and a special inflationary solution with constant internal volume. In particular it was shown that inflationary solutions in the Brans-Dicke frame transform into non-inflationary ones in the Einstein frame. It is to be expected that this is a rather general feature, whence the multitude of solutions which appear inflationary in the Brans-Dicke frame will be indeed non-inflationary when considered in the Einstein frame.

\section{Discussion}

The Einstein action with minimally coupled scalars and $p$-branes in higher dimension $D$ can be reduced to an effective model in lower dimension $D_{0}$. This results in a (generalized) $\sigma$-model with conformally flat target space. With a purely geometrical dilaton field $f$, it provides a natural generalization for the well-known Brans-Dicke theory.

The orthobrane condition (5.6) allows us to find exact solutions. Furthermore, the orthobrane solution is a sufficient condition for the target space of the $\sigma$-model to be a locally symmetric space. The orthobrane case is the generic one (apart from cases with degenerate coupling matrix (3.17)) where the target space is locally symmetric.

Examples of a certain minimal static, spherically symmetric $p$-brane configuration are given with just one electric and one magnetic antisymmetric $F$ component (since in 4 dimensions we only deal with a single electromagnetic field), which in general intersect and interact with a single scalar field. Spherical symmetry here is considered in the physical relevant $D_{0}=4$ case of $S^{2}$ spheres, although the extension to arbitrary spheres is straightforward.

Besides popular families of orthobrane solutions there are further families of solutions, which have another additional symmetry, e.g. coinciding $F$-field charges for the electro-magnetic solutions. In the target space this additional symmetry is expressed by a linear dependency (8.34) between column vectors $Y$ of the coupling matrix $L$ defined in (3.17).

For the mentioned static solutions, Hawking temperature $T_{H}$ can be formally calculated by 
surface gravity via a Komar-like integral. For both, the orthobrane case and the case of equal charges $Q_{e}^{2}=Q_{m}^{2}$, the expressions of $T_{H}$ depend characteristically on the intersection dimension. This results are also interesting in the context of recent increased interest in extremal $p$-brane configurations with black holes [24].

The interpretation of the extremal limit $k \rightarrow 0$ is delicate. The solutions above have been described in isotropic coordinates which cover just the asymptotically flat exterior of the black hole. A better understanding of their global causality structure would require an investigation of the maximal extension of the space-time rather than only of its exterior part. The limit $k \rightarrow 0$ was here called extremal, since via (8.29) and (8.48) in this limit the effective asymptotical Schwarzschild mass $M$ is just given by the charges, $G_{N} M=\frac{1}{2}\left(B p_{\mathrm{e}}+C p_{\mathrm{m}}\right)$ and $G_{N} M=p /\left(1+d_{2}\right)$, respectively. Further work is required to understand this type of extremality, and the related asymptotics of $T_{H}$, which remains finite for intersection dimension $d_{2}=1$ and becomes infinite for $d_{2} \geq 2$. As it was pointed out recently in [25] particular care is needed in order to associate the correct physical charges and thermal properties of a black hole correctly with its horizon.

The multidimensional $\sigma$-model opens the door for further investigations, in particular also for covariant and canonical quantization. The effective $\sigma$-model reduction appears as a possible clue to canonical quantization within a large well defined class of higher dimensional geometries, namely the multidimensional ones. Covariant quantization techniques can be applied in any dimension $D_{0}$. In particular, they are well applicable to our new solutions with scalar fields only, when the target space is flat. The effective geometry of the $D_{0}$-dimensional model can be reformulated in terms of connections and soldering forms. For $D_{0}=4$, not only a canonical $1+3$ split can be performed, but moreover the canonical quantization of the $D_{0^{-}}$-geometry can be performed with self-dual variables in the usual manner.

Finally, in analogy to investigations in [4], it should be possible to apply solution generating techniques like the Ehlers-Harrison transformation also in the context of the multidimensional $\sigma$-model.

\section{Acknowledgments}

I would like to thank to A. Ashtekar, O. Brodbeck, K. Bronnikov, S. Das, L. Freidel, S. Gupta, V. Ivashchuk, J. Pullin, R. Puzio, and L. Smolin for discussions on the subject, and to N. Guerras for permanent encouragement. This work was supported by NSF grant PHY-9514240 to The Pennsylvania State University and a gift from the Jesse Phillips Foundation. 


\section{References}

[1] P. Breitenlohner, D. Maison, and G. Gibbons, Commun. Math. Phys. 120, 295-333 (1988).

[2] R. Geroch, J. Math. Phys. 12, 918-924 (1971); J. Math. Phys. 13, 394-404 (1972).

[3] D.V. Gal'tsov, Phys. Rev. Lett. 74, 2863-2866 (1995).

[4] D.V. Gal'tsov and O.A. Rytchkov, Generating branes via sigma-models, hep-th/9801160.

[5] D. Korotkin and H. Samtleben, Yangian symmetry in integrable quantum gravity, hepth/9710210.

[6] A. Strominger and C. Vafa, Phys. Lett. B 379, 99 (1996).

[7] M. J. Duff, Int. J. Mod. Phys. A 11, 5623 (1996).

[8] J. Scherk and J. H. Schwarz, Phys. Lett. B 57, 463 (1975).

[9] J.K. Beem, P.E. Ehrlich, and K.L. Easley, Global Lorentzian Geometry, Pure and Applied Mathematics 202, 2nd. Edition, Marcel Dekker Inc.,NY (1996)

[10] M. Rainer and A. Zhuk, Phys. Rev. D 54, 6186 (1996).

[11] G. W. Gibbons and S. W. Hawking, Phys. Rev. D 15, 2752 (1977).

[12] J. W. York, Phys. Rev. Lett. 28, 1082 (1972); Found. Phys. 16, 249 (1986).

[13] M. Rainer, Multidimensional Scalar-Tensor Theories and Minisuperspace Approach, publ. in: Hadronic Journal 21, 351 (1998); Proc. Int. Workshop on Modern Modified Theories of Gravitation and Cosmology, (Beer Sheva, June 29-30, 1997).

[14] M. Rainer, Int. J. Mod. Phys. D 4, 397 (1995).

[15] M. Rainer, Gravitation \& Cosmology 1, 121 (1995).

[16] H. Bondi and T. Gold, Month. Not. R. Astron. Soc. 108, 252 (1948).

[17] U. Bleyer and A. Zhuk, Astron. Nachr. 317, 161 (1996).

[18] V. D. Ivashchuk, V. N. Melnikov, and M. Rainer. "Multidimensional $\sigma$-models with composite electric p-branes". gr-qc/9705005, publ. in: Gravitation \& Cosmology 4, No. 1, 73-82 (1998). 
[19] K. A. Bronnikov, U. Kasper, and M. Rainer. Intersecting electric and magnetic p-branes: spherically symmetric solutions. Preprint P-Math-97/21, gr-qc/9708058.

[20] M. Heusler, Black hole uniqueness theorems, Cambridge Lecture Notes in Physics 6, Cambridge University Press (1996).

[21] U. Bleyer and A. Zhuk, Gravitation \& Cosmology 1, 37 (1995); ibid 1, 106 (1995).

[22] M. Gasperini and G. Veneziano, Mod. Phys. Lett. A 8, 3701 (1993); Phys. Rev. D 50, 2519 (1994).

[23] M. Rainer and A. Zhuk, Einstein and Brans-Dicke frames in multidimensional cosmology, gr-qc/9808073.

[24] Y. Satoh, BTZ black holes and theit near-horizon geometry of higher-dimensional black holes, hep-th/9810135

[25] A. Ashtekar, J. Baez, A. Corichi, K. Krasnov, Phys.Rev.Lett. 80, 904-907 (1998) 